\title{
Generalizations of entanglement based on coherent states and convex sets
}

\author{
Howard Barnum, 周 Emanuel Knill, fi] Gerardo Ortiz, f and Lorenza Viola \\ Los Alamos National Laboratory, MS B256, Los Alamos, NM 87545
}

(Dated: October 25, 2018)

\begin{abstract}
Unentangled pure states on a bipartite system are exactly the coherent states with respect to the group of local transformations. What aspects of the study of entanglement are applicable to generalized coherent states? Conversely, what can be learned about entanglement from the well-studied theory of coherent states? With these questions in mind, we characterize unentangled pure states as extremal states when considered as linear functionals on the local Lie algebra. As a result, a relativized notion of purity emerges, showing that there is a close relationship between purity, coherence and (non-)entanglement. To a large extent, these concepts can be defined and studied in the even more general setting of convex cones of states. Based on the idea that entanglement is relative, we suggest considering these notions in the context of partially ordered families of Lie algebras or convex cones, such as those that arise naturally for multipartite systems. The study of entanglement includes notions of local operations and, for information-theoretic purposes, entanglement measures and ways of scaling systems to enable asymptotic developments. We propose ways in which these may be generalized to the Lie-algebraic setting, and to a lesser extent to the convex-cones setting. One of our motivations for this program is to understand the role of entanglement-like concepts in condensed matter. We discuss how our work provides tools for analyzing the correlations involved in quantum phase transitions and other aspects of condensed-matter systems.
\end{abstract}

\footnotetext{
* barnum@lanl.gov

thnill@lanl.gov

g_ortiz@lanl.gov

lviola@lanl.gov
} 


\section{Contents}

I. Introduction $\quad 3$

II. Entanglement for Bipartite Quantum Systems 5

A. Characterizing Product States $\quad 5$

B. Local Quantum Maps

C. Communication Complexity 13

D. Resource Scaling 13

E. Measures of Entanglement $\quad 14$

III. The Lie-algebraic Setting 16

A. Purity, Coherence and Entanglement 17

B. Local Quantum Maps 19

C. Communication Complexity 21

D. Resource Scaling $\quad 22$

E. Measures of Relative Entanglement 22

F. Other Measures 23

\begin{tabular}{ll}
\hline IV. The Convex Cones Setting & 24
\end{tabular}

\begin{tabular}{ll}
\hline A. Convex Cones & 24 \\
\hline
\end{tabular}

B. Local Maps 25

\begin{tabular}{ll}
\hline C. Measures of Relative Entanglement & 27
\end{tabular}

D. Monotonicity for Explicit Liftable Maps 27

\begin{tabular}{lr}
\hline V. Discussion & 29
\end{tabular}

$\begin{array}{ll}\text { A. Further Examples and Extensions } & 29\end{array}$

B. Relevance to Condensed Matter Physics $\quad 30$

C. Conclusion $\quad 32$

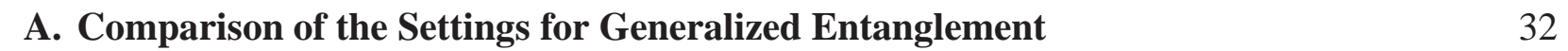

$\begin{array}{ll}\text { Acknowledgments } & 35\end{array}$

$\begin{array}{ll}\text { References } & 35\end{array}$ 


\section{INTRODUCTION}

Entangled states are joint states of two or more distinguishable quantum systems that cannot be expressed as a mixture of products of states of each system. Entangled states can exhibit quantum correlations between the two systems that have no local classical interpretation. One of the most important developments in the study of quantum mechanics was the characterization of these correlations by Bell [1], 2], whose many experimental verifications [3], 勾] (see also [5] and the references therein) have given further support to the validity of quantum mechanics. Entangled states are now widely considered to be the defining resource of quantum communication, enabling protocols such as quantum teleportation [6] and leading to great improvements in the communication efficiency of certain multi-party tasks [7, 8]. As a result, entanglement is being actively investigated both from a physical and from an information-theoretic perspective.

So far, nearly all studies of entanglement involved two or more distinguishable quantum subsystems. As a result, investigations of entanglement have focused on understanding how quantum systems are made up from subsystems and how this differs from classical systems. However, there are a number of signs that the assumption of distinguishable quantum subsystems is too narrow to capture all properties of states that one might like to ascribe to entanglement. Several authors [9, 10, 11, 12, 13, 14, 15, 16, 17] have considered entanglement-related notions for bosons and fermions. For example, consider the state of one photon in two coupled cavities. Being the state of one particle, there is a tendency to expect that there is no entanglement, because one particle cannot be entangled. On the other hand, each cavity is a quantum system. From the point of view of these two quantum systems, the state where the photon is in an equal superposition of being in either cavity can be represented as $(|10\rangle+|01\rangle) / \sqrt{2}$ and is clearly entangled. Another example involving photons is provided by optical "cat states" [18, 19]. In this case, cat states are quantum superpositions of sufficiently distinct coherent states in a mode. As the name suggests, such states are thought to involve entanglement. They certainly have distinctive non-classical behavior, but since they exist in a single system (the mode) the strict interpretation of entanglement based on subsystems would indicate that no entanglement is present. A third example is that consisting of a number of fermions in a lattice. The "simple" states for such a system are described by the so-called Slater determinants (see, for example, [20], p. 7), which describe the wavefunction of noninteracting fermions. Because the fermions in such a wavefunction are independent, one expects that no entanglement is present in such a state. However, from the point of view of the lattice modes, most Slater determinants exhibit entanglement [21]. The three examples make it clear that the presence or absence of entanglement depends on the physically relevant point of view. Here we propose that this point of view depends on the relationships between different Lie algebras of observables that determine the dynamics and our ability to control the system of interest. In particular, the extent to which entanglement is present depends on the observables used to measure a system and describe its states.

One of our goals is to show that the relationships between product states, separable states and entangled states are at least Lie-algebraic in nature, and to some extent even more general. This makes it possible to study the salient features of entanglement without reference to subsystems, using instead whatever Lie algebras are physically relevant. For the case of bipartite quantum systems, the relevant Lie algebra $\mathfrak{h}$ consists of the unilocal operators (operators of the form $A \otimes$ $I$ or $I \otimes B)$. To show that the ideas of entanglement, separability and product states do not critically depend on the two subsystems, we provide several ways in which product states can be characterized in terms of $\mathfrak{h}$ alone. All of these ways lead to the same concept for general semisimple Lie algebras, namely that of generalized coherent states [22, 23, 24]. It is therefore 
natural to consider product states to be special kinds of coherent states. From this perspective, separable states are mixtures of coherent states, and pure entangled states are incoherent pure states. Another way to think about these structures is to realize that the coherent states are exactly those states which are relatively pure, that is, extremal with respect to the set of expectations of observables in the Lie algebra. Thus, pure states are entangled if they appear to be mixed with respect to the Lie algebra's expectations. In the case of bipartite quantum systems, this is an aspect of entanglement that has long been considered a key nonclassical property of quantum mechanics: Pure entangled states have mixed reduced density operators whereas, for example, in classical probability no pure state can have a mixed marginal. See, for example, [25], p. 298, [26], p. 116 and [27], p. 306.

The recognition that incoherence naturally generalizes entanglement makes explicit the dependence of the notion of entanglement on the relevant Lie algebra and makes available the tools of the theory of generalized coherent states [23, 24] for investigating aspects of entanglement. To extend the power of this perspective to the information-theoretic applications of entanglement requires introducing measures of entanglement, generalizing the ways in which entanglement can be manipulated and providing a means for using states as a resource. In bipartite systems, there is an abundance of measures of entanglement, many of which generalize naturally. Further measures arise naturally in the general context and specialize to potentially interesting measures for multipartite systems that have not yet been considered. In bipartite systems, a key role is played by LOCC (local quantum operations and classical communication) maps. We propose several classes of maps for general semisimple Lie algebras that, in the case of bipartite systems, are related to LOCC. A desirable property of entanglement measures is that they are monotone non-increasing under LOCC. We can show monotonicity properties for some classes of maps in the general setting. To introduce the notion of states as a resource and enable asymptotic analysis, we consider schemes for associating Lie algebras with tensor products of systems defined by a given representation of a semisimple Lie algebra.

For the purpose of determining what are the essential properties of states needed to study entanglement, we introduce a setting even more general than Lie algebras. Since the states when viewed as linear functionals on observables form a convex cone, we generalize the definitions to the setting where we have two or more convex cones related by positive maps. The cones represent the family of states as linear functionals on the Lie algebras. In the case of bipartite systems, these are the local Lie algebra and the Lie algebra of all operators. The map relating the two state spaces is the restriction map of linear functionals. The definitions relating to separability and entanglement only require this structure. Entanglement measures can also be defined based only on convexity, and so can various notions of local maps.

In taking seriously the idea that entanglement is a relative notion, one finds that in many cases, there are many more than two relevant Lie algebras. In the bipartite case, we can consider the hierarchy of algebras consisting of the trivial Lie algebra, the algebra of operators acting on the first system, that acting on the second system, the sum of these, and the algebra of all operators. When there are more than two systems, the number of different ways of combining local Lie algebras multiplies. For photons, there is the Lie algebra of passive linear operations, of active linear operations, and that of all linear and nonlinear operations. To these one might add the Lie algebras acting locally on the modes, etc. It is in the increasing amount of information that is available about states as more operators are added that crucial quantum properties emerge. We believe that in studying a given system, it is beneficial to consider coherence and entanglement properties at multiple levels.

Independently of the work reported here, Klyachko [28] has recently proposed a generalization 
of entanglement for representations of semisimple Lie groups. His starting point is an extremality property that we use as one of the equivalent characterizations of product (in general, coherent) states. Klyachko's work is focused on the geometric invariant theory approach for investigating states with respect to one Lie group of operators. This approach leads to useful classifications of the orbits of states under the Lie group's action. In this context, he discusses how the notions of classical realism that lead to Bell's inequalities [1] generalize to the Lie algebraic setting. He also introduces notions of maximal entanglement and another interesting entanglement measure.

In Section II, we introduce the basic notions required for generalizing separability and entanglement by reviewing the example of bipartite systems from the point of view of Lie algebras and coherence. The generalization to semisimple Lie-algebras is explained in Section III, and the extent to which the generalization depends only on the relationships between convex cones is discussed in Section IV. For reference, the different settings for studying entanglement are compared in Table A. The paper concludes with a discussion of other relevant examples and the potential applications to condensed matter. We assume familiarity with the basic concepts of quantum information and entanglement. A good reference for quantum information theory is [29]. For reviews of entanglement, see [30, 31, 32]. We also use results from the basic theory of Lie algebras. Details can be found in books such as [33, 34, 35, 36]. For physically motivated treatments of Lie algebras, see [37, 38, 39, 40, 41]. References for convexity and convex cones include [42, 43].

\section{ENTANGLEMENT FOR BIPARTITE QUANTUM SYSTEMS}

The standard setting for studying entanglement involves two (or more) distinguishable quantum subsystems forming a bipartite system. The properties of entanglement are most salient if the quantum subsystems are spatially well separated, with communication between the sites restricted to classical signals subject to speed-of-light limitations. Let the state space of two such quantum subsystems be given by the Hilbert spaces $\mathcal{H}_{a}$ and $\mathcal{H}_{b}$ of dimension $N_{a}$ and $N_{b}$, respectively. The joint state space of the bipartite system is $\mathcal{H}_{a b}=\mathcal{H}_{a} \otimes \mathcal{H}_{b}$. All state spaces and operator algebras are assumed to be finite dimensional. See Section $\mathrm{VA}$ for a brief discussion of the need and possibilities for extensions to infinite dimensional systems. Product states are pure states of $\mathcal{H}_{a b}$ of the form $|\psi\rangle \otimes|\phi\rangle$. Entangled pure states are states of $\mathcal{H}_{a b}$ that are not expressible as a product state. It is necessary to generalize the state space to mixtures of pure states, that is probability distributions over pure states. For this purpose, one uses density matrices to represent states. A density matrix $\rho$ is pure if $\rho=|\psi\rangle\langle\psi|$ for some $|\psi\rangle$. Equivalently, it is pure if $\operatorname{tr}\left(\rho^{2}\right)=1$, or if $\rho$ is extremal in the set of density matrices (see below). A separable state is a mixture of product states. Its density matrix is therefore a convex combination of product states, which is a sum of the form $\sum_{k} p_{k}\left|\psi_{k}\right\rangle\left\langle\psi_{k}|\otimes| \phi_{k}\right\rangle\left\langle\phi_{k}\right|$, where $\left(p_{k}\right)_{k}$ is a probability distribution [44]. We will use the expressions "convex combination" and "mixture" interchangeably. A non-separable state is said to be entangled. It is worth recalling that separable states can have non-classical features. For example, see [45, 46].

\section{A. Characterizing Product States}

In our approach, the key distinction between entangled and separable states is the difference between the way things look locally and globally. The local observables are operators of the form $A \otimes I$ and $I \otimes B$. For our purposes, it is convenient to allow arbitrary operators as observables, not only hermitian ones. Since non-hermitian operators can be expressed as complex linear 
combinations of hermitian operators, expectations of such operators are readily computed from expectations of hermitian operators.

If a pure state of the two systems in unentangled, then it is completely determined by the expectation values of the local observables. To specify a pure entangled state requires knowledge of the correlations, which are expectations of operators of the form $A \otimes B$. Note that this method for distinguishing between unentangled and entangled states does not extend to mixtures. A generic separable state can contain non-trivial correlations. An example is $(|0\rangle\langle 0|\otimes| 0\rangle\langle 0|+| 1\rangle\langle 1|\otimes| 1\rangle\langle 1|) / 2$. Here the two subsystems are classically correlated. Nevertheless, it is possible to characterize separability by investigating the structure of states in terms of their expectations of local versus global observables.

There are four non-trivial Lie algebras of observables that determine the structure of the bipartite system. Let $\mathfrak{h}_{a}\left(\mathfrak{h}_{b}\right)$ be the Lie algebra of operators of the from $A \otimes I(I \otimes B)$ acting on system $a$ (b). We call these the unilocal algebras, because they consist of operators acting on one subsystem only. The local Lie algebra is given by $\mathfrak{h}_{l}=\mathfrak{h}_{a}+\mathfrak{h}_{b}$. Let $\mathfrak{g}$ be the Lie algebra of all operators on $\mathcal{H}_{a b}$. As defined, these four Lie algebras are complex. However, as families of operators they are $\dagger$-closed, that is, closed under hermitian conjugation. Let $\operatorname{Re}(\mathfrak{h})$ be the set of hermitian operators in $\mathfrak{h}$. For a hermitian-closed space of operators $\mathfrak{h}, \mathfrak{h}=\operatorname{Re}(\mathfrak{h})+i \operatorname{Re}(\mathfrak{h})$, where $i=\sqrt{-1}$. Using complex Lie algebras simplifies the representation theory and is useful for defining generalizations of local quantum maps (see Section [I B). Although exponentials $e^{A}$ for non-skew-hermitian operators are not unitary, they can be interpreted as Lie-algebraically definable operators associated with postselected outcomes in an implementation of a quantum map.

A simple way of characterizing product states without referring to the underlying partition into two subsystems can be based on unique ground states. A unique ground state of a hermitian operator is a unique minimum-eigenvalue eigenstate. Operators with degenerate minimum-eigenvalue eigenspaces do not have a unique ground state. In general, we call the the minimum-eigenvalue eigenspace of an operator the ground space.

Theorem $1|\psi\rangle \in \mathcal{H}_{a b}$ is a product state iff it is the unique ground state of an operator in $\operatorname{Re}\left(\mathfrak{h}_{l}\right)$.

Proof: Suppose that $|\psi\rangle$ is the unique ground state of $H=A \otimes I+I \otimes B \in \operatorname{Re}\left(\mathfrak{h}_{l}\right)$. The ground space of $H$ is the intersection of the ground spaces of $A \otimes I$ and $I \otimes B$, which are product subspaces. Thus, a unique ground state is a product state. Conversely, let $|\psi\rangle=\left|\phi_{a}\right\rangle \otimes\left|\phi_{b}\right\rangle$. Choose an operator $A(B)$ on $\mathcal{H}_{a}\left(\mathcal{H}_{b}\right)$ such that $\left|\phi_{a}\right\rangle\left(\left|\phi_{b}\right\rangle\right)$ is the unique ground state of $A(B)$. Then $|\psi\rangle$ is the unique ground state of $A \otimes I+I \otimes B \in \operatorname{Re}\left(\mathfrak{h}_{l}\right)$.

We can use Theorem 11 to define a generalization of a product state for any hermitian-closed Lie algebra of operators. As we will see in Section III, this generalization agrees with the notion of generalized coherent states.

The distinction between product and entangled states can also be viewed in terms of purity with respect to the relevant algebra of operators. It can be seen that product states are exactly the states whose reduced density matrices on each of the two subsystems are pure. The two reduced density matrices for a state completely determine the expectations of the observables in the local Lie algebra. To prepare for generalizing these observations, consider states as linear functionals on the Lie algebras in question. We define an $\mathfrak{h}$-state to be a linear functional $\lambda$ on the operators of $\mathfrak{h}$ induced by a density matrix $\rho$ according to $\lambda(C)=\operatorname{tr}(\rho C)$. The set of $\mathfrak{h}$-states is denoted by $\mathfrak{h}^{+}$. In the present setting, states are completely determined by the linear functional on the Lie algebra of all operators $\mathfrak{g}$ induced by their density matrix. A $\mathfrak{g}$-state $\lambda$ can be restricted to each of the Lie algebras $\mathfrak{h}_{a}, \mathfrak{h}_{b}$ and $\mathfrak{h}_{l}$. For example, the restriction $\lambda \mid \mathfrak{h}_{a}$ of $\lambda$ to $\mathfrak{h}_{a}$ determines the expectations of 
observables on the first subsystem, and therefore the reduced density matrix associated with the state.

Consider the set $\mathfrak{h}_{l}^{+}$of $\mathfrak{h}_{l}$-states. This set is closed under convex (or probabilistic) combination. That is, if the $\lambda_{k}$ are $\mathfrak{h}_{l}$-states, then so is $\sum_{k} p_{k} \lambda_{k}$ for any probability distribution $\left(p_{k}\right)_{k}$. By compactness, all states in $\mathfrak{h}_{l}^{+}$can be obtained as convex combinations of extremal states (or extreme points of $\mathfrak{h}_{l}^{+}$). Extremal states are states not expressible as a convex combination of other states. If the only information available about a state are the expectations of observables in $\mathfrak{h}_{l}$, then states that induce extremal expectations, that is, extremal elements of $\mathfrak{h}_{l}^{+}$, are those about which there is the least uncertainty. It therefore makes sense to call such states pure, or $\mathfrak{h}_{l}$-pure, to be specific.

Theorem 2 An $\mathfrak{h}_{l}$-state is pure iff it is induced by a pure product state.

Proof: Consider a density matrix $\rho$ inducing the $\mathfrak{h}_{l}$-state $\lambda$. The state $\lambda$ is determined by the reduced density matrices of $\rho$. It is possible to find a probabilistic combination of pure product states with the same reduced density matrices, which therefore also induces $\lambda$. This implies that every $\mathfrak{h}_{l}$-state is expressible as a probabilistic combination of $\mathfrak{h}_{l}$ states induced by pure product states. Consequently, the pure $\mathfrak{h}_{l}$-states are among those induced by pure product states. Conversely, if $\lambda$ is not pure, then $\lambda$ can be nontrivially expressed in the form $p \lambda_{1}+(1-p) \lambda_{2}$ where the $\lambda_{k}$ are $\mathfrak{h}_{l}$-states. It follows that the two reduced density matrices that can be deduced from $\lambda$ are not both pure: They are mixtures of the reduced density matrices deduced from $\lambda_{k}$, and since $\lambda_{1} \neq \lambda_{2}$, at least one of these mixtures is nontrivial.

The previous theorem shows that the difference between pure unentangled states and pure entangled states is that as expectations of $\mathfrak{h}_{l}$, the latter are not extremal. If the only information that is available are expectations of $C \in \mathfrak{h}_{l}$, it is not possible to distinguish between entangled states and unentangled mixed (that is, separable) states. To distinguish, we need expectations of other operators. It is worth noting what it means to have access only to expectations of sets of observables. Given only a single instance of a quantum system, the expectations cannot be inferred. On the other hand, with sufficiently powerful control, it is possible to realize a projective measurement of the eigenvalues of observables, a process that gives information not just about the expectation of an observable, but also about the expectations of its powers. One situation where access to expectations only is realistic is when the quantum system must be accessed collectively in large ensembles involving mostly identical states. In an appropriate weak interaction and large ensemble limit, the effect on other large systems reveals the expectations of observables involved in the interaction, whereas the effect on the systems in the ensemble tends to a unitary evolution with the observable as a Hamiltonian. The weak interaction therefore naturally limits the available control to Lie algebras generated by a small number of observables. An example where this situation occurs for systems that are best modeled as being quantum is nuclear magnetic resonance of molecules in the liquid state [47].

\section{B. Local Quantum Maps}

One can compare states in the context of information processing resources by considering families of "local" quantum maps that can be used to convert states. For bipartite systems, as well as for multipartite systems in general, the most important such family, LOCC, consists of maps that can be implemented with local quantum maps with access to ancillas and classical communication (see [29], Sect. 12.5). A larger family, the separable quantum maps, have an operator-sum representation consisting of operators of the form $A \otimes B$. Separable quantum maps are readily 
generalized to the Lie algebraic setting, whereas we have not yet found an equally convincing generalization of LOCC.

A quantum map is a trace-preserving completely-positive linear transformation of density operators. Rather than define these terms, we use the fact that every quantum map can be written in the operator-sum representation as $\rho \rightarrow \sum_{k} C_{k} \rho C_{k}^{\dagger}$, with $\sum_{k} C_{k}^{\dagger} C_{k}=I$. We will also consider completely-positive maps, which have the same form, but don't require the constraint on the $C_{k}$. To define LOCC, we make the sequence $\mathbf{C}=\left(C_{k}\right)_{k}$ explicit and define $\mathbf{C}(\rho)=\sum_{k} C_{k} \rho C_{k}^{\dagger}$. Note that the sequence $\mathbf{C}$ is not uniquely determined by the map. We call $\mathbf{C}$ an explicit map. See [29], $\mathrm{p}$. 372 for how to determine when two explicit maps act the same. To avoid trivial degeneracies, we assume that the operators that define an explicit map are always non-zero. If $\left(\mathbf{D}_{k}\right)_{k}$ is a sequence of explicit quantum maps, then the conditional composition of $\mathbf{C}$ and $\left(\mathbf{D}_{k}\right)_{k}$ is the quantum map with operator sequence $\left(D_{k l} C_{k}\right)_{k l}$ and action $\rho \rightarrow \sum_{k l} D_{k l} C_{k} \rho C_{k}^{\dagger} D_{k l}^{\dagger}$. A unilocal quantum map is a map of the form $\left(A_{k} \otimes I\right)_{k}$ or $\left(I \otimes B_{k}\right)_{k}$. LOCC is the set of quantum maps obtained as conditional compositions of unilocal maps. The length of the composition is associated with the number of rounds of classical communication. A separable map is a completely positive map with an explicit form given by $\left(A_{k} \otimes B_{k}\right)_{k}$. Note that all LOCC maps are necessarily separable. The set of separable maps has been called SLOCC [48, 49, 50] and can be viewed as maps that can be implemented with LOCC and postselection based on the communication record.

Quantum maps as defined here are often called "quantum operations" [29], though the latter term is sometimes extended to include non-trace-preserving completely positive maps. In this manuscript, we use the word "map" to refer to linear functions of spaces other than the Hilbert space of the quantum system under consideration. We use the word "operator" to refer to linear functions from the Hilbert space to itself. An important role in defining various notions of local maps is played by explicit maps, which in the bipartite and in the Lie algebraic setting are completely-positive by definition. There is the potential for confusion in referring to explicit maps. For example, an explicit map can be separable without the operators in the explicit representation having the necessary product form. To simplify the terminology, we position the adjective "explicit" such that it applies to all modifiers between it and the word "map". For example, an explicit separable map $\mathbf{C}=\left(C_{k}\right)_{k}$ satisfies that each $C_{k}$ is a product operator, whereas this is not required of separable explicit maps.

Separable maps can be defined from $\mathfrak{h}_{l}$ without reference to the two component subsystems.

Theorem 3 A completely positive map is separable iff it has an explicit representation $\left(C_{k}\right)_{k}$ with $C_{k} \in \overline{e^{\mathfrak{h}_{l}}}$.

By definition, $\overline{e^{\mathfrak{h}_{l}}}$ is the topological closure of the set of all exponentials of operators in $\mathfrak{h}_{l}$. The notion of closure may be based on the norm induced by the matrix inner product $\operatorname{tr}\left(A^{\dagger} B\right)$.

Proof: $e^{\mathfrak{h}_{l}}$ consists of all non-zero determinant operators of the form $A \otimes B$. Thus $e^{\mathfrak{h}_{l}}$ contains all invertible product operators, which are dense in the set of product operators. The set of product operators is closed.

There are separable quantum maps that are not LOCC [45]. The goal is to define or construct, with minimal reference to the two subsystems, quantum maps that respect locality better than separable ones. For example, in order to construct the family of LOCC maps, it is sufficient to be able to determine when an operator in $\mathfrak{h}_{l}$ is unilocal, and when a family of unilocal operators all act on the same side. With this ability, one can construct LOCC as was done above, by conditional composition. If the ability does not depend on the bipartite nature of the system, there is hope that LOCC has a non-trivial generalization. 
We have two approaches to obtaining families of separable quantum maps with stronger locality properties. The first approach is based on the observation that unilocal maps induce well-defined transformations of $\mathfrak{h}_{a^{-}}, \mathfrak{h}_{b^{-}}$and $\mathfrak{h}_{l^{-}}$-states. To formally define what this means, let $\mathbf{C}$ be an explicit map. Then $\mathbf{C}$ acts on the set of linear functionals $\mathfrak{g}^{*}$ of $\mathfrak{g}$ according to $\mathbf{C}(\lambda)(X)=\lambda\left(\sum_{k} C_{k}^{\dagger} X C_{k}\right)$. It will be clear from context whether we are applying $\mathbf{C}$ to operators or to linear functionals. The map C, but not its explicit form, is determined by the action on $\mathfrak{g}$-states. Note also that $\mathfrak{g}$-states linearly span all linear functionals on $\mathfrak{g}$, and similarly for $\mathfrak{h}$ states with $\mathfrak{h}$ one of $\mathfrak{h}_{a}, \mathfrak{h}_{b}$ or $\mathfrak{h}_{l}$. C induces a well-defined transformation of $\mathfrak{h}$-states if we can complete the following commutative diagram with a map $\mathbf{C}^{\prime}$ of $\mathfrak{h}^{*}$ :

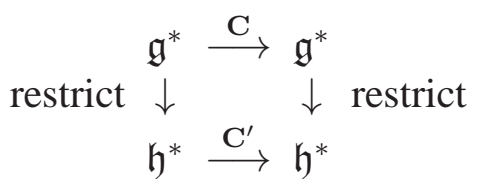

where $\mathfrak{h}^{*}$ is the set of linear functionals on $\mathfrak{h}$. Equivalently, whenever $\lambda_{1}$ and $\lambda_{2}$ are $\mathfrak{g}$-states that agree on $\mathfrak{h}$, that is, for which $\lambda_{1} \uparrow \mathfrak{h}=\lambda_{2} \uparrow \mathfrak{h}$, it is the case that $\mathbf{C}\left(\lambda_{1}\right) \mathfrak{h}=\mathbf{C}\left(\lambda_{2}\right) \mathfrak{h}$. Equivalently, if

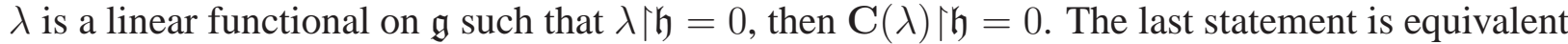
to the statement that $\mathbf{C}$ preserves the nullspace of the restriction map. If any of the above properties hold, we say that $\mathbf{C}$ can be lifted to $\mathfrak{h}$. Its lifting is the map $\mathbf{C}^{\prime}$ induced on $\mathfrak{h}$-states.

In the present setting, the notion of liftability can be simplified by using the canonical (via the trace inner product) isomorphism $\mu$ between $\mathfrak{h}^{*}$ and $\mathfrak{h}$. Because the trace inner product is non-degenerate when restricted to the $\dagger$-closed set of operators $\mathfrak{h}$, the isomorphism $\mu$ is uniquely determined by the identity $\lambda(C)=\operatorname{tr}\left(\mu(\lambda)^{\dagger} C\right)$ for all $C \in \mathfrak{h}$. In particular, for the algebra $\mathfrak{g}$ of all operators on $\mathcal{H}_{a b}$, if $\lambda \in \mathfrak{g}^{*}$ is induced by the operator $X$, then $\mu(\lambda)=X$. In general, we say that the linear functional $\lambda$ is induced by $\mu(\lambda)$. Let $\operatorname{tr}_{a}\left(\operatorname{tr}_{b}\right)$ denote the partial trace mapping operators on $\mathcal{H}_{a b}$ to operators on $\mathcal{H}_{b}\left(\mathcal{H}_{a}\right.$, respectively). We have the following identities:

$$
\begin{aligned}
\mu\left(\lambda \uparrow \mathfrak{h}_{a}\right) & =\operatorname{tr}_{b}(\mu(\lambda)) \otimes I / N_{b}, \\
\mu\left(\lambda\left\lceil\mathfrak{h}_{b}\right)\right. & =I / N_{a} \otimes \operatorname{tr}_{a}(\mu(\lambda)), \\
\mu\left(\lambda \mid \mathfrak{h}_{l}\right) & =\operatorname{tr}_{b}(\mu(\lambda)) \otimes I / N_{b}+I / N_{a} \otimes \operatorname{tr}_{a}(\mu(\lambda))-\operatorname{tr}(\mu(\lambda))(I \otimes I) /\left(N_{a} N_{b}\right) .
\end{aligned}
$$

These identities witness the fact that the reduced density matrices of a state determine the induced linear functionals on the local Lie algebras. In the range of $\mu$, the nullspaces of the restriction maps to $\mathfrak{h}_{a}, \mathfrak{h}_{\mathfrak{b}}$, and $\mathfrak{h}_{l}$ correspond to the spaces spanned by $A \otimes B$ with $B, A$ and both $A$ and $B$, respectively, traceless. Using the fact that product operators are a basis of all operators on $\mathcal{H}_{a} \otimes \mathcal{H}_{b}$, it can be seen that the explicit map $\mathbf{C}$ lifts to $\mathfrak{h}_{a}$ iff $\operatorname{tr}_{b} \sum_{k} C_{k}(A \otimes B) C_{k}^{\dagger}=\mathbf{C}^{\prime}(A) \operatorname{tr}(B)$ for some map $\mathbf{C}^{\prime}$. Equivalently, it lifts iff whenever $\operatorname{tr}(B)=0$, then $\operatorname{tr}_{b} \sum_{k} C_{k}(A \otimes B) C_{k}^{\dagger}=0$. Similar statements can be made about $\mathfrak{h}_{b}$. C lifts to $\mathfrak{h}_{l}$ iff whenever both $\operatorname{tr}(A)=0$ and $\operatorname{tr}(B)=0$, then $\operatorname{tr}_{b} \sum_{k} C_{k}(A \otimes B) C_{k}^{\dagger}=0$ and $\operatorname{tr}_{a} \sum_{k} C_{k}(A \otimes B) C_{k}^{\dagger}=0$.

Most completely positive maps, even LOCC ones, cannot be lifted. An example for two qubits is the "conditional reset" map that first measures qubit $a$, and if the measurement outcome is $|1\rangle$, it resets qubit $b$ to $|0\rangle$. However, the unilocal maps are liftable. In fact, they are liftable to both $\mathfrak{h}_{a}$ and $\mathfrak{h}_{b}$, as are (unconditional) compositions of unilocal maps. This is the case because such maps are determined by their actions on the reduced density matrices. This suggests that liftable explicit quantum maps could be used as a generating set for quantum maps with more locality then separable quantum maps. We next discuss some of the properties of liftable separable maps and their relationship to LOCC. 
Theorem 4 Let $\mathbf{C}=\left(C_{1} \otimes C_{2}\right)$ be a one-operator, explicit separable map liftable to $\mathfrak{h}_{l}$. Then $C=\alpha U \otimes V$ with $U$ and $V$ unitary.

Proof: Liftability implies that if $A$ and $B$ are traceless, then $C_{1} A C_{1}^{\dagger}$ and $C_{2} B C_{2}^{\dagger}$ are traceless. This implies that the map $f: A \rightarrow C_{1} A C_{1}^{\dagger}$ satisfies that $\operatorname{tr}(f(A))=\alpha_{1} \operatorname{tr}(A)$ for some $\alpha_{1}$. Thus $f / \alpha_{1}$ is trace preserving, from which it follows that $C_{1}^{\dagger} C_{1}=\alpha_{1} I$. For the same reason, $C_{2}^{\dagger} C_{2}=\alpha_{2} I$. The conclusion of the theorem now follows, with $\alpha=\sqrt{\left|\alpha_{1} \alpha_{2}\right|}$.

Theorem $\mathbf{5}$ Let $\mathbf{C}$ be an explicit separable map that lifts to the identity map on $\mathfrak{h}_{b}$. Then $\mathbf{C}$ is unilocal, acting on system a only.

Proof: Write $\mathbf{C}=\left(D_{k} \otimes E_{k}\right)_{k}$, where $D_{k} \otimes E_{k} \neq 0$ for all $k$. By assumption and applying the map to $I \otimes B$,

$$
\sum_{k} \operatorname{tr}\left(D_{k} D_{k}^{\dagger}\right) E_{k} B E_{k}^{\dagger}=N_{a} B
$$

If for some $k, E_{k} \not \subset I$, we can find $|\psi\rangle\langle\psi|$ such that $E_{k}|\psi\rangle\langle\psi| E_{k}^{\dagger}$ 's one-dimensional range does not contain $|\psi\rangle$. Because for all $l, \operatorname{tr}\left(D_{l} D_{l}^{\dagger}\right)>0$, the left side of Equation 2 also has this property, contradicting the identity. Hence $E_{k}=\alpha_{k} I$ for each $k$ and the result follows.

Theorem 5 characterizes unilocal maps but has the disadvantage that we have to refer explicitly to the unilocal Lie algebras, thus requiring more information about locality than that provided by $\mathfrak{h}_{l}$ alone. This suggests the following problem:

Problem 6 Are separable quantum maps that lift to $\mathfrak{h}_{l}$ LOCC? Are they LOCC if they lift to both $\mathfrak{h}_{a}$ and $\mathfrak{h}_{b}$ ?

If the answer to this problem is "no", then we are interested in the question of whether the explicit separable quantum maps that are liftable to $\mathfrak{h}_{l}$ generate all separable quantum maps by conditional composition.

In order to be able to conditionally compose explicit separable quantum maps that are LOCC without departing from LOCC, we need the explicit representations to have the additional property that they can be LOCC implemented in such a way that the communication record reveals which of the operators in the sequence occurred. Following our convention for using the adjective "explicit", we call an explicit quantum map with this property an explicit LOCC map.

Problem 7 Are there explicit separable quantum maps that are LOCC but not explicit LOCC?

If the answers to this problem and to Problem 6 are "yes", then one has to consider the strengthening of the questions in Problem 6 where "separable" is replaced by "explict separable" and "LOCC" by "explicit LOCC". This is required so that conditional composition can be used without leaving LOCC. Here is one case where we can prove that a family of quantum maps is explicit LOCC.

Theorem 8 Let $\mathbf{C}=\left(D_{k} \otimes E_{k}\right)$ be an explicit separable quantum map that lifts to $\mathfrak{h}_{l}$ with the additional property that $\left(D_{k}^{\dagger} D_{k}\right)_{k}$ is linearly independent. Then $E_{k}=\gamma_{k} U_{k}$ with $U_{k}$ unitary. In particular, $\mathrm{C}$ is an explicit LOCC map. 
Proof: Using the identification of linear functionals with operators, consider linear functionals $\lambda$ of $\mathfrak{g}$ induced by $A \otimes B$ with $\operatorname{tr}(B)=0$. The restriction of $\lambda$ to $\mathfrak{h}_{l}$ is induced by $\operatorname{tr}(A) I \otimes B \in \mathfrak{h}_{l}$. The restriction has only scalar dependence on $A$. Restricting after applying $\mathbf{C}$ gives the linear functional induced by

$$
\sum_{k} \operatorname{tr}\left(D_{k} A D_{k}^{\dagger}\right) I / N_{a} \otimes E_{k} B E_{k}^{\dagger}+\sum_{k} D_{k} A D_{k}^{\dagger} \otimes \operatorname{tr}\left(E_{k} B E_{k}^{\dagger}\right) I / N_{b}
$$

Note that because $\operatorname{tr}(B)=0$, and the assumption that the map is trace preserving, the contribution to $I \otimes I$ vanishes. Because of liftability, the same scalar dependence applies to this expression. By cyclicity of the trace, $\operatorname{tr}\left(D_{k} A D_{k}^{\dagger}\right)=\operatorname{tr}\left(A D_{k}^{\dagger} D_{k}\right)$. Because the $D_{k}^{\dagger} D_{k}$ are independent, we can choose $A_{l}$ such that $\operatorname{tr}\left(A_{l} D_{k}^{\dagger} D_{k}\right)=\delta_{l k}$. Hence the following are all scalar multiples of the same operator, where the scalar is independent of $B$ :

$$
O_{l}=I / N_{a} \otimes E_{l} B E_{l}^{\dagger}+\sum_{k} D_{k} A_{l} D_{k}^{\dagger} \otimes \operatorname{tr}\left(E_{k} B E_{k}^{\dagger}\right) I / N_{b}
$$

Computing the partial trace over the first system, we get

$$
\begin{aligned}
\operatorname{tr}_{a}\left(O_{l}\right) & =E_{l} B E_{l}^{\dagger}+\sum_{k} \operatorname{tr}\left(A_{l} D_{k}^{\dagger} D_{k}\right) \operatorname{tr}\left(B E_{k}^{\dagger} E_{k}\right) / N_{b} & & \text { by cyclicity of trace, } \\
& =E_{l} B E_{l}^{\dagger}+\sum_{k} \operatorname{tr}\left(\left(A_{l} \otimes B\right)\left(D_{k}^{\dagger} D_{k} \otimes E_{k}^{\dagger} E_{k}\right)\right) / N_{b} & & \text { because tr is multiplicative for } \otimes, \\
& =E_{l} B E_{l}^{\dagger}+\operatorname{tr}\left(A_{l} \otimes B\right) / N_{b} & & \text { because } \mathrm{C} \text { is a quantum map, } \\
& =E_{l} B E_{l}^{\dagger} & & \text { because } \operatorname{tr}(B)=0 .
\end{aligned}
$$

Consequently, the operators $E_{l} B E_{l}^{\dagger}$ are all proportional with constant of proportionality independent of $B$. Consider $E=E_{r}$. We have

$$
E_{l} B E_{l}^{\dagger}=\alpha_{l} E B E^{\dagger}
$$

for all traceless $B$, where $\alpha_{l} \operatorname{tr}\left(A_{r}\right)=\operatorname{tr}\left(A_{l}\right)$. Reformulating, we get that for all traceless $B$, $\operatorname{tr}\left(B E_{l}^{\dagger} E_{l}\right)=\alpha_{l} \operatorname{tr}\left(B E^{\dagger} E\right)$. Hence $E_{l}^{\dagger} E_{l}=\alpha_{l} E^{\dagger} E+\beta_{l} I$ for some $\beta_{l}$. The trace-preserving condition requires that

$$
\begin{aligned}
I \otimes I & =\sum_{k} D_{k}^{\dagger} D_{k} \otimes E_{k}^{\dagger} E_{k} \\
& =\sum_{k} D_{k}^{\dagger} D_{k} \otimes\left(\alpha_{k} E^{\dagger} E+\beta_{k} I\right) \\
& =\left(\sum_{k} \alpha_{k} D_{k}^{\dagger} D_{k}\right) \otimes E^{\dagger} E+\left(\sum_{k} \beta_{k} D_{k}^{\dagger} D_{k}\right) \otimes I
\end{aligned}
$$

Suppose that the traceless part of $E^{\dagger} E$ is not zero, Then $\sum_{k} \alpha_{k} D_{k}^{\dagger} D_{k}=0$, which is possible only if $\alpha_{k}=0$ for all $k$ (by independence). But by construction $\alpha_{r}=1$, so $E^{\dagger} E$ is a multiple of the identity, hence $E=E_{r}$ is a multiple of a unitary operator, say $E_{r}=\gamma_{r} U_{r}$. Returning to the trace-preserving condition (Equation 6 ) and using the fact that $r$ was arbitrary, we find that $\sum_{k} \gamma_{k} D_{k}^{\dagger} D_{k} \bar{\gamma}_{k}=I$. This makes $\mathbf{D}=\left(\gamma_{k} D_{k} \otimes I\right)_{k}$ a unilocal quantum map. The $U_{k}$ can be implemented conditionally on which $D_{k}$ occurs in a unilocal implementation of $\mathbf{D}$, hence $\mathbf{C}$ is LOCC. 
Corollary 9 Let $\mathbf{C}=\left(D_{1} \otimes E_{1}, D_{2} \otimes E_{2}\right)$ be an explicit separable quantum map that lifts to $\mathfrak{h}_{l}$. Then $\mathrm{C}$ is explicit LOCC.

Proof: The result follows by Theorem 8 unless $D_{2}^{\dagger} D_{2}=\alpha^{\prime} D_{1}^{\dagger} D_{1}$ and $E_{2}^{\dagger} E_{2}=\beta^{\prime} E_{1}^{\dagger} E_{1}$ for some $\alpha$ and $\beta$. In this case, using the trace-preserving condition, $D_{1}^{\dagger} D_{1} \otimes E_{1}^{\dagger} E_{1} \propto I \otimes I$ making all operators proportional to unitaries. Such an map can be realized explicitly with LOCC by first creating a shared random variable, then implementing local unitaries conditional on the random variable.

Every explicit unilocal quantum map can be obtained as a composition of binary unilocal quantum maps, where a binary quantum map is an explicit quantum map consisting of two operators. The modifier "explicit" is assumed when using the modifier "binary". We can therefore use the corollary to characterize LOCC as the quantum maps obtained by conditional composition of binary separable quantum maps that lift to $\mathfrak{h}_{l}$.

Instead of using liftability as the basis for generalizing LOCC and other classes of local maps, one can use the spectral properties of the constituent operators of an explicit quantum map. This idea is motivated by the following result:

Theorem 10 An operator in $\operatorname{Re}\left(\mathfrak{h}_{l}\right)$ that has a maximal ground space is unilocal.

Maximal means maximal among ground spaces different from $\mathcal{H}$ of operators in $\operatorname{Re}\left(\mathfrak{h}_{l}\right)$.

Proof: An operator in $\operatorname{Re}\left(\mathfrak{h}_{l}\right)$ is of the form $A \otimes I+I \otimes B$. By subtracting a multiple of the identity, we can assume that $A$ and $B$ traceless, not both zero. If they are both non-zero, then the operator's ground space is strictly contained in that of $A \otimes I$, hence not maximal.

For future reference, an operator whose traceless part is zero or satisfies the condition of Theorem 10 is said to be maximally unilocal. Note that except for $N_{a}=N_{b}=2$, not all unilocal operators in $\operatorname{Re}\left(\mathfrak{h}_{l}\right)$ are maximally unilocal. However, two maximally unilocal operators $C_{1}$ and $C_{2}$ with ground spaces $H_{1}$ and $H_{2}$ such that $H_{2}=e^{D} H_{1}$ for some $D \in \mathfrak{h}_{l}$ act on the same side. Also, if $C_{1}$ is maximally unilocal and $C_{2}=e^{D} C_{1} e^{-D}$ with $D \in \mathfrak{h}_{l}$, then $C_{2}$ is unilocal and acts on the same side. We call a family of operators contained in the span of $\left\{e^{D} C e^{-D} \mid D \in \mathfrak{h}_{l}\right\}$ with $C$ maximally unilocal an m-compatible unilocal family. With this definition, we have:

Theorem 11 An explicit unilocal quantum map consists of an m-compatible unilocal family of operators.

Proof: Every unilocal one-dimensional projector is maximally unilocal, and the span of the conjugates under $e^{\mathfrak{h}_{l}}$ of one such projector consists of all operators acting on the same side.

Using this theorem, we can characterize LOCC as the set of quantum maps obtained by conditional composition of explicit m-compatible quantum maps. However, this characterization is not directly related to the definition of separable maps. To do so requires introducing explicit quantum maps whose operators are exponentials of members of an m-compatible family. Also note that in addition to using linear closure in the definition of m-compatibility, we could have used closure under commutators (Lie bracket). In the bipartite setting, this makes no difference. Alternatively, we could have left out linear closure and just used conjugation under $e^{\mathfrak{h}_{l}}$. We do not know whether conditional composition of the resulting quantum maps yields LOCC. See the discussion of this topic in Section IIIB. 


\section{Communication Complexity}

In the study of multiparty protocols, an important issue is the communication complexity of converting one state to another using LOCC maps. The communication complexity is defined as the number of classical bits that need to be communicated. The communication complexity of a particular LOCC map to a given state can be determined from a representation as a conditional composition. This can be done by adding the resources used in each round. The contribution from a round depends on the previous map in the sequence of conditional compositions, as we now explain. Suppose that the initial state's density matrix is given by $\rho$, the total explicit quantum map before the round under consideration is $\mathbf{C}$, and this is then conditionally composed with the family of unilocal explicit quantum maps $\mathbf{B}_{k}$. In general, given an explicit quantum map $\mathbf{D}$ applied to density matrix $\rho$, the average number of bits needed to communicate the outcomes is given by $H(\mathbf{D}, \rho)=-\sum_{k} p_{k} \log p_{k}$, where $p_{k}=\operatorname{tr}\left(\rho D_{k}^{\dagger} D_{k}\right)$ is the probability of outcome $D_{k}$. This is of course an asymptotic expression assuming knowledge of $\rho$. In other cases one might prefer to just use $\log |\mathbf{D}|$ as the number of bits required. In any case, the contribution to the communication complexity of the current round is the average communication complexity for transmitting the information in the outcomes of the conditionally applied maps. This quantity is given by

$$
\sum_{k} \operatorname{tr}\left(\rho C_{k}^{\dagger} C_{k}\right) H\left(\mathbf{B}_{k}, C_{k} \rho C_{k}^{\dagger} / \operatorname{tr}\left(\rho C_{k}^{\dagger} C_{k}\right)\right)
$$

The contributions from each round are added up to obtain the communication complexity of the sequence of conditional compositions. Depending on the application, the contribution of the last round can be omitted as its outcomes need not be communicated to implemented the quantum map. Note that if the detailed outcomes in one round are not required for conditioning in the next rounds, then the explicit maps can be modified to defer these outcomes until the last round, which is one reason to omit the contribution of the last round.

In general, the goal is to implement a given communication task with (near) minimum communication complexity. By determining the complexity according to Expression 8, we can generalize communication complexity to any scheme for defining a family of quantum maps by conditional composition, including the generalized local maps to be introduced for the Lie algebraic setting in Section III.

\section{Resource Scaling}

An important aspect of information theory involves asymptotic characterizations of the relationships between information resources and of the complexity of tasks. To asymptotically scale up a problem, one usually creates tensor copies of the bipartite states involved and then investigates their relationships in the context of the now much larger bipartite system. The relationship between the local Lie algebras of the individual bipartite subsystems and the one obtained after forming the tensor products requires a construction other than the usual products. We did not find an obvious way of implementing such a construction that does not rely on knowledge of additional structure. It may be the case that one must have knowledge of how the representation of $\mathfrak{h}_{l}$ was constructed. Nevertheless, there are a few things we can say that may help in better understanding how resources can be scaled and how to implement asymptotic analyses.

We construct the space $\mathcal{H}=\mathcal{H}_{a b} \otimes \ldots \otimes \mathcal{H}_{a b}$ as an $n$-fold tensor product of copies of $\mathcal{H}_{a b}$. Let $\mathfrak{h}_{l, k}$ be the local Lie algebra acting on the $k$ 'th factor. Let $\mathfrak{h}_{L}$ be the local Lie algebra for $\mathcal{H}$, where 
$\mathcal{H}$ is bipartitioned into $\mathcal{H}_{a} \otimes \ldots \otimes \mathcal{H}_{a}$ and $\mathcal{H}_{b} \otimes \ldots \otimes \mathcal{H}_{b}$. Define $\mathfrak{h}_{a, k}, \mathfrak{h}_{A}, \mathfrak{h}_{b, k}$ and $\mathfrak{h}_{B}$ likewise. The group of permutations on $n$ elements acts on $\mathcal{H}$ by permuting the tensor factors. The goal is to establish how $\mathfrak{h}_{L}$ relates to the $\mathfrak{h}_{l, k}$. It suffices to consider the case $n=2$, because we can view $\mathfrak{h}_{L}$ as the smallest Lie algebra that contains the appropriate Lie algebras obtained for each pair of factors.

Let $G_{2}\left(\mathfrak{h}_{x}\right)(x \in\{a, b, l\})$ be the set of operators $C$ on $\mathcal{H}_{a b} \otimes \mathcal{H}_{a b}$ such that for all operators $X$ on $\mathcal{H}_{a b}, \operatorname{tr}_{1}\left(C\left(X^{\dagger} \otimes I\right)\right) \in \mathfrak{h}_{x, 2}$ and $\operatorname{tr}_{2}\left(C\left(I \otimes X^{\dagger}\right)\right) \in \mathfrak{h}_{x, 1}$. Here, $\operatorname{tr}_{i}$ is tracing out the $i$ 'th factor with respect to the tensor product $\mathcal{H}_{a b} \otimes \mathcal{H}_{a b}$. In words, $G_{2}\left(\mathfrak{h}_{x}\right)$ is the set of operators which look locally like operators in $\mathfrak{h}_{x}$.

Theorem $12 G_{2}\left(\mathfrak{h}_{a}\right)=\mathfrak{h}_{A}, G_{2}\left(\mathfrak{h}_{b}\right)=\mathfrak{h}_{B}$, but $G_{2}\left(\mathfrak{h}_{l}\right)$ strictly contains $\mathfrak{h}_{L}$.

Proof: The definition ensures that $\mathfrak{h}_{A} \subseteq G_{2}\left(\mathfrak{h}_{a}\right)$. Let $C \in G_{2}\left(\mathfrak{h}_{a}\right)$. We can write $C=$ $\sum_{k l r s} \alpha_{k l r s}\left(A_{k} \otimes B_{l}\right) \otimes\left(A_{r} \otimes B_{s}\right)$ with $\left(A_{k}\right)_{k}$ and $\left(B_{l}\right)_{l}$ orthonormal bases of operators including the identity. The ordering of the tensor product is according to $\left(\mathcal{H}_{a} \otimes \mathcal{H}_{b}\right) \otimes\left(\mathcal{H}_{a} \otimes \mathcal{H}_{b}\right)$. Suppose that $\alpha_{k l_{0} r s}$ is non-zero for some $l_{0}$ with $B_{l_{0}} \neq I$. Then using $X=A_{r} \otimes B_{s}$ in the definition of $G_{2}$ and tracing out we get $\sum_{k l} \alpha_{k l r s} A_{k} \otimes B_{l}$, which is not in $\mathfrak{h}_{a}$ due to the term $B_{l_{0}}$. By symmetry, this establishes the first two identities. The third statement follows from the observation that any operator of the form $(A \otimes I) \otimes(I \otimes B)$ is in $G_{2}\left(\mathfrak{h}_{l}\right)$. If $A$ and $B$ are traceless, this operator is not in $\mathfrak{h}_{L}$.

The above theorem provides ways of constructing $\mathfrak{h}_{A}$ and $\mathfrak{h}_{B}$ but not $\mathfrak{h}_{L}$. However, one can construct $\mathfrak{h}_{L}$ as the Lie algebra generated by $\mathfrak{h}_{A}$ and $\mathfrak{h}_{B}$. This depends on the bipartition only through its emergence from having the two unilocal Lie algebras.

Another way in which one can attempt to construct $\mathfrak{h}_{X}$ involves using a group of unitary operators that extends the permutations group $S_{n}$ acting on the factors. $S_{n}$ by itself is insufficient, in the sense that the Lie algebra generated by $g C g^{\dagger}$ for $g$ a permutation operator and $C \in \mathfrak{h}_{x, k}$ is just $\bigoplus_{k} \mathfrak{h}_{x, k}$. A sufficiently large extension suffices. An example is the group $U \otimes V$, with $U$ and $V$ acting on the tensor products of the $\mathcal{H}_{a}$ and $\mathcal{H}_{b}$ factors, respectively, which generates $\mathfrak{h}_{X}$ from $\mathfrak{h}_{x, 1}$ by conjugation. The problem is whether such an extension can be chosen naturally. An idea that does not work but might have some independent interest is to consider the Lie algebra $\mathfrak{h}_{X}^{\prime}$ generated by $g C g^{\dagger}$ with $C \in \mathfrak{h}_{x, 1}$ and $g$ a unitary operator in the group algebra generated by the permutation operators. To see that this does not yield the desired Lie algebras, let $s$ be the swap operator. Then $g=(I+i s) / \sqrt{2}$ is unitary, but $g((A \otimes I) \otimes(I \otimes I)) g^{\dagger}$ is not in $\mathfrak{h}_{A}$.

\section{E. Measures of Entanglement}

For pure states $|\psi\rangle$ of a bipartite system the generally accepted and information-theoretically meaningful measure of entanglement is given by the von Neumann entropy of either one of the reduced density matrices for $|\psi\rangle$ [51]. Thus, the entanglement of $|\psi\rangle$ can be computed as the Shannon entropy of the spectrum of the reduced density matrix on the first (or, equivalently, the second) system. For $\mathfrak{h}_{l}$-states, the underlying Hilbert space is not directly accessible. However, there are natural complexity measures associated with the convex structure of these states. To define such measures, let $S$ be a Schur-concave function of probability distributions. By definition, Schur-concave functions are permutation invariant and concave (see for example [52], pp. 40). That is, if $\mathbf{p}$ and $\mathbf{q}$ are two probability distributions of the same length where the probabilities of $\mathbf{q}$ are a permutation of those of $\mathbf{p}$, then $S(\mathbf{p})=S(\mathbf{q})$; and if $\mathbf{p}=r \mathbf{p}_{1}+(1-r) \mathbf{p}_{2}$ for $r \geq 0$, then $S(\mathbf{p}) \geq r S\left(\mathbf{p}_{1}\right)+(1-r) S\left(\mathbf{p}_{2}\right)$. An example of a Schur-concave function is the Shannon 
entropy. For a pure state $|\psi\rangle$ define $S(|\psi\rangle)$ to be $S$ evaluated on the spectrum of the reduced density matrices. For an $\mathfrak{h}_{l}$-state $\lambda$, define

$$
S(\lambda)=\inf \left\{S(\mathbf{p}) \mid \lambda=\sum_{k} p_{k} \lambda_{k} \text { with } \lambda_{k} \mathfrak{h}_{l} \text {-pure }\right\} .
$$

We will routinely overload the function $S$. Which definition is intended is communicated through the argument. So far, the argument type can be a probability distribution, a state in $\mathcal{H}_{a b}$ or an $\mathfrak{h}_{l}$-state.

Theorem 13 If the $\mathfrak{h}_{l}$-state $\lambda$ is induced by a pure state $|\psi\rangle$ on the bipartite system, then $S(\lambda)=$ $S(|\psi\rangle)$.

Proof: Using the Schmidt decomposition, we can write $|\psi\rangle=\sum_{k} \sqrt{p_{k}}\left|\phi_{k}\right\rangle \otimes\left|\varphi_{k}\right\rangle$ with $\left(\left|\phi_{k}\right\rangle\right)_{k}$ and $\left(\left|\varphi_{k}\right\rangle\right)_{k}$ orthonormal bases and $S(|\psi\rangle)=S(\mathbf{p})$. If the $\lambda_{k}$ are the pure $\mathfrak{h}_{l}$-states induced by $\left|\phi_{k}\right\rangle \otimes\left|\varphi_{k}\right\rangle$, then $\lambda=\sum_{k} p_{k} \lambda_{k}$. It follows that $S(\lambda) \leq S(|\psi\rangle)$.

To prove that $S(\lambda) \geq S(|\psi\rangle)$, write $\lambda=\sum_{k} p_{k} \lambda_{k}$, with $\lambda_{k} \mathfrak{h}_{l}$-pure and $S(\mathbf{p})$ arbitrarily close to $S(\lambda)$. To be specific, $S(\mathbf{p}) \leq S(\lambda)+\epsilon$. By Theorem [2], the $\lambda_{k}$ are pure product states. Let $\lambda_{k}$ be induced by $\left|\phi_{k}\right\rangle \otimes\left|\varphi_{k}\right\rangle$. Define $\rho=\sum_{k} p_{k}\left|\phi_{k}\right\rangle\left\langle\phi_{k}|\otimes| \varphi_{k}\right\rangle\left\langle\varphi_{k}\right|$. Then $\operatorname{tr}_{b}(\rho)=\sum_{k} p_{k}\left|\phi_{k}\right\rangle\left\langle\phi_{k}\right|$ and is equal to the corresponding reduced density matrix for $|\psi\rangle$. It therefore suffices to prove that $S(\mathbf{p})$ is at least $S$ evaluated on the spectrum of $\rho_{a}$. One way to see this it is to write $\rho_{a}=A P A^{\dagger}$, where $A$ consists of unit-length columns (the $\left|\phi_{k}\right\rangle$ ) and $P$ is the diagonal matrix with the $p_{k}$ 's on the diagonal. The eigenvalues of $\rho_{a}$ are the same as those of $P^{1 / 2} A^{\dagger} A P^{1 / 2}$. This matrix has the $p_{k}$ on the diagonal. The result now follows from the fact that $\mathbf{p}$ is a transformation of the spectrum by a doubly stochastic matrix (see, for example, [29], page 513), doubly stochastic matrices are convex combinations of permutation matrices (see, for example, [53], page 36; [29], page 574), and concavity of $S$.

Theorem 13 makes it possible to introduce entanglement measures without reference to the underlying pair of systems while being faithful to the known measures for such systems. We extend the entanglement measure $S$ to mixed states by a second minimization over convex representations as pure states [54]. To do so, consider a $\mathfrak{g}$-state $\lambda$ induced by the density matrix $\rho$. With respect to the convex set of $\mathfrak{g}$-states, $\lambda$ is pure iff $\rho$ is pure. The distinction between separability and entanglement can be seen to be one associated with the purity of a state from the points of view of $\mathfrak{g}$ and $\mathfrak{h}_{l}$. Thus, we define

$$
S\left(\lambda ; \mathfrak{h}_{l}\right)=\inf \left\{\sum_{k} p_{k} S\left(\lambda_{k} \mid \mathfrak{h}_{l}\right) \mid \lambda=\sum_{k} p_{k} \lambda_{k} \text { with } \lambda_{k} \mathfrak{g} \text {-pure }\right\}
$$

Because of the isomorphism between density matrices $\rho$ and $\mathfrak{g}$-states, this expression defines an entanglement measure for arbitrary bipartite density matrices. In anticipation of the generalizations to come, we explicitly introduced the Lie algebra $\mathfrak{h}_{l}$ as a parameter.

Suppose that $S(\mathbf{p})=0$ iff $\mathbf{p}$ is pure, that is, $p_{k}=\delta_{j k}$ for some $j$. We call such an $S$ proper. Then a $\mathfrak{g}$-state $\lambda$ satisfies $S\left(\lambda ; \mathfrak{h}_{l}\right)=0$ iff it is a mixture of product states, which justifies thinking of $S$ as an entanglement measure. Several properties are desirable of an entanglement measure [51]. For example, the measure should be convex and it should be non-increasing under LOCC maps. Both of these properties are satisfied by $S$ as defined above [48].

Entanglement measures can be based on asymptotic convertibility of states with respect to a family of local maps. For example, one can define $R(\rho, \sigma)$ as the asymptotic supremum of $r / s$, 
where $r$ is the number of asymptotically good copies of $\rho$ that can be constructed from $s$ copies of $\sigma$ given any number of additional product states and using separable quantum maps. For more precise definitions of this sort, see [55]. If there is a reasonable choice $\sigma$ of a maximally entangled state, then $R(\rho, \sigma)$ can be considered to be an entropy of formation and $R(\sigma, \rho)$ an entropy of distillation. By varying the constraints on the quantum maps different measures are obtained.

\section{THE LIE-ALGEBRAIC SETTING}

To generalize the notions introduced in the previous section requires not much more than removing the connection between the local Lie algebra and the bipartite system. As a consequence we will learn that product states are generalized coherent states.

We fix a finite dimensional Hilbert space $\mathcal{H}\left(\mathcal{H}_{a b}\right.$ in the bipartite setting) and consider states from the point of view of various $\dagger$-closed, complex Lie algebras of operators acting on $\mathcal{H}$. Ultimately, we consider families of Lie algebras $\left(\mathfrak{h}_{x}\right)_{x}$ acting on $\mathcal{H}$ and ordered by inclusion. But first we consider one $\dagger$-closed Lie algebra $\mathfrak{h}$. By default we assume that $I$ is a member of our operator Lie algebras. The set of traceless operators of $\mathfrak{h}$ is denoted by $\mathfrak{h}_{\circ}$. The abstract Lie algebra faithfully represented by $\mathfrak{h}$ is denoted by $\mathfrak{h}$. The assumption that $\mathfrak{h}$ is $\dagger$-closed implies that $\breve{h}$ is reductive (see, for example, [56], Sect. 1.7). A reductive Lie algebra $\mathfrak{r}$ is one that consists of the direct product of an abelian $\mathfrak{a}$ and a semisimple Lie algebra $\mathfrak{s}$ (see, for example, [56], Sect. 1.7, or [33], p. 102). The direct product is in the category of Lie algebras and homomorphisms of Lie algebras and corresponds, after exponentiation, to the direct product of groups. In this case it means that as vector spaces, $\mathfrak{r}=\mathfrak{a} \oplus \mathfrak{s}$, where $\mathfrak{a}$ commutes with $\mathfrak{s}$. For Lie algebras, $x$ and $y$ commute iff $[x, y]=0$. A semisimple Lie algebra is one which is a direct product of simple Lie algebras, where a simple Lie algebra is one that is not abelian and has no proper ideals. Reductiveness of our Lie algebras is useful because the finite-dimensional semisimple Lie algebras and their representations have been completely classified (see, for example, [33]). If $\mathfrak{h}$ is irreducible as a set of operators, then the abelian part consists only of multiples of the identity operator, and the semisimple part consists of the traceless operators.

The two examples for $\mathfrak{h}$ to keep in mind are $\mathfrak{h}_{l}$ in the bipartite setting and the set of generators of the spatial rotations of a spin- 1 particle. In the second example, the Hilbert space is three dimensional with basis $|-1\rangle,|0\rangle$ and $|1\rangle$ corresponding to the three states with definite spin along $z$. The Lie algebra $\mathfrak{h}$ is spanned by the identity together with the spin operators $J_{z}, J_{x}$ and $J_{y}$. The corresponding abstract Lie algebra is $1 \times \mathfrak{s l}_{2} \mathbb{C}$, where 1 is the one-dimensional Lie algebra. As linear spaces, this is the same as $1 \oplus \mathfrak{s l}_{2} \mathbb{C}$, the operator $\times$ emphasizes the fact that the construction is a direct product, so that the two Lie algebras commute. For this example we take $\mathfrak{g}$ to consist of all operators.

Before proceeding, we recall the basic properties of semisimple Lie algebras that are needed to define generalized coherent states and relate them our characterizations of product states in the bipartite setting.

A Cartan subalgebra of $\mathfrak{h}_{\circ}$ is a maximal abelian subalgebra whose elements are diagonalizable (that is, semisimple). According to a fundamental result for Lie algebras, Cartan subalgebras exist and are conjugate (hence isomorphic) with respect to an operator in $e^{\text {ho }_{0}}$ ([33], pp. 81-87; [35], Thm. D.22, p. 492; [34], Thm. 4.1.2, p. 263). Every diagonalizable operator in $\mathfrak{h}$ is contained in some Cartan subalgebra. If the operator is hermitian, the Cartan subalgebra can be chosen to be $\dagger$-closed. Let $\mathfrak{c}$ be a Cartan subalgebra of $\mathfrak{h}_{0}$, then $\mathcal{H}$ can be decomposed into the joint eigenspaces for $\mathfrak{c}, \mathcal{H}=\bigoplus_{\alpha} \mathcal{H}_{\alpha}$, where the $\alpha$ are distinct linear functionals on $\mathfrak{c}$ such that for $|\psi\rangle \in \mathcal{H}_{\alpha}$ and $A \in \mathfrak{c}, A|\psi\rangle=\alpha(A)|\psi\rangle$. ([33], p. 107; [35], p. 199 eq. (14.4)). The $\mathcal{H}_{\alpha}$ are called the weight 
spaces for $\mathfrak{c}$ and the $\alpha$ are called the weights. In general, a weight for a Cartan subalgebra is a linear functional for which there exists a finite dimensional representation with a non-empty corresponding weight space. The abstract Lie algebra $\mathfrak{h}_{\circ}$ can be represented on itself by the Lie bracket. This is called the adjoint representation of $\check{\mathfrak{h}}_{\circ}$. The weights for this representation are called roots. It turns out that the geometrical properties of the roots determine the Lie algebra. The roots are in effect also linear functionals on $\mathfrak{c}$. There are special sets of roots called simple root systems (or bases) that span the linear functionals on $\mathfrak{c}$ and have the property that every root is either a positive, or a negative integral combination of simple roots. The former are called positive roots. The definition depends on the choice of simple roots, but not in a crucial way, because all simple root systems are isomorphic via a special kind of isomorphism (a member of the so-called Weyl group, [33], p. 51; [35], Prop. D.29, p. 494). The weights can be partially ordered by defining $\alpha \leq \alpha^{\prime}$ if $\alpha^{\prime}-\alpha$ is a positive integral sum of simple roots. With this ordering, in an irreducible representation, there is a unique minimum weight, whose weight space is onedimensional ([33], pp. 108-109; [35], Prop. 14.13, pp. 202-203). The minimum weight state depends on the choice of Cartan subalgebra and simple roots. However, $e^{i \operatorname{Re}\left(\mathfrak{h}_{\mathfrak{b}}\right)}$ acts transitively on the set of minimum weight vectors. Furthermore, every minimum weight vector can be obtained by means of a $\nmid$-closed Cartan subalgebra of $\mathfrak{h}$. The minimum weight space has the property that it is annihilated by operators in $\mathfrak{h}_{0}$ which are in root spaces associated with negative roots. In fact, this is another characterization of the minimum weight space (see the definition and theorem in [33], p. 108). Usually, treatments of semisimple Lie algebras focus on the maximum weights of a representation. Here we choose to use the equivalent minimum weights because of the relationship to ground states of Hamiltonians. The basic properties of Cartan subalgebras and the notions of roots and weights extend from semisimple to reductive Lie algebras by adjoining the abelian part.

A family of generalized coherent states consists of an orbit of a dynamical group acting on a state space [23, 24]. According to this definition, every state is in a family of generalized coherent states. As a result, an important part of the theory of generalized coherent states is to choose those orbits that best generalize the properties of the coherent states familiar in optics. In our case, the dynamical groups are Lie groups generated by semisimple Lie algebras. If the goal is to choose states that are in a sense the most classical, then there are strong arguments for choosing the minimum weight states of a representation of the Lie group. Theorem 14 below provides some of these arguments. We therefore use the term generalized coherent state, or simply coherent state, to refer specifically to minimum weight states of a Lie algebra. Because we only consider finite dimensional representations, our treatment does not directly apply to the conventional coherent states of optics, for example. In this case, the relevant Lie algebra is the Heisenberg algebra, which is not semisimple (or reductive). The standard †-closed representation is therefore necessarily infinite. The theory of coherent states suggests that extensions to such Lie algebras and representations are possible [23].

\section{A. Purity, Coherence and Entanglement}

For a $\nmid$-closed Lie algebra of operators $\mathfrak{h}$ on $\mathcal{H}$, define $\mathfrak{h}$-states as before as linear functionals on $\mathfrak{h}$ induced by a state's density matrix $\rho$ according to $\lambda(A)=\operatorname{tr}(\rho A)$. Observe again that the set $\mathfrak{h}^{+}$of $\mathfrak{h}$-states is convex closed. Pure $\mathfrak{h}$-states are extreme points of $\mathfrak{h}^{+}$. Suppose that the $\mathfrak{h}$-state $\lambda$ is induced by the density matrix $\rho$. We can project $\rho$ onto $\mathfrak{h}$ with respect to the trace inner product. Denote the projection map onto $\mathfrak{h}$ by $\mathcal{P}_{\mathfrak{h}}$. Because $\mathfrak{h}$ is $\nmid$-closed, the projection $\mathcal{P}_{\mathfrak{h}}(\rho)$ is a hermitian operator in $\mathfrak{h}$. Furthermore, $\lambda$ is also induced by $\mathcal{P}_{\mathfrak{h}}(\rho)$, that is, $\lambda(A)=\operatorname{tr}\left(P_{\mathfrak{h}}(\rho) A\right)=\operatorname{tr}(\rho A)$ for $A \in \mathfrak{h}$. Note that in general, $\mathcal{P}_{\mathfrak{h}}(\rho)$ is not positive. For example, let $\rho$ be the density matrix for $|1\rangle$ 
in the spin-1 example. Another important observation is that $\mathcal{P}_{\mathfrak{h}}(\rho)$ depends only on $\lambda$. That is, if $\rho$ and $\rho^{\prime}$ both induce $\lambda$, then $\mathcal{P}_{\mathfrak{h}}(\rho)=\mathcal{P}_{\mathfrak{h}}\left(\rho^{\prime}\right)$.

We now assume that $\mathfrak{h}$ acts irreducibly on $\mathcal{H}$. If it does not act irreducibly, decompose $\mathcal{H}$ into irreducible invariant subspaces for $\mathfrak{h}$ and consider each of these subspaces separately. Define the $\mathfrak{h}$-purity of $\lambda$ as $\operatorname{tr}\left(\mathcal{P}_{\mathfrak{h}}(\rho)^{2}\right)$, where $\lambda$ is induced by the density matrix $\rho$. This is of course the length of $\mathcal{P}_{\mathfrak{h}}(\rho)$ according to the trace-inner-product norm. The $\mathfrak{h}$-purity is bounded above by the conventional purity $\operatorname{tr}\left(\rho^{2}\right)$, which is the $\mathfrak{g}$-purity with $\mathfrak{g}$ the algebra of all operators on $\mathcal{H}$. This generalization of purity is useful because according to Theorem 14 below, the pure $\mathfrak{h}$-states are exactly the states with maximum $\mathfrak{h}$-purity.

The goal of the remainder of this subsection is to give a number of useful characterizations of pure $\mathfrak{h}$-states. In particular, we show that they are exactly the coherent states for $\mathfrak{h}$. We first state the characterization theorem and then discuss the equivalent characterizations before proving the theorem.

Theorem 14 The following are equivalent for a density matrix $\rho$ inducing the $\mathfrak{h}$-state $\lambda$ :

(1) $\lambda$ is a pure $\mathfrak{h}$-state.

(2) $\rho=|\psi\rangle\langle\psi|$ with $|\psi\rangle$ the unique ground state of some $H$ in $\operatorname{Re}(\mathfrak{h})$.

(3) $\rho=|\psi\rangle\langle\psi|$ with $|\psi\rangle$ a minimum-weight vector (for some simple root system of some Cartan subalgebra) of $\mathfrak{h}_{\mathrm{o}}$.

(4) $\lambda$ has maximum h-purity.

(5) $\rho$ is a one-dimensional projector in $\overline{e^{\mathfrak{h}}}$.

This theorem is a synthesis of various largely known results in the representation theory of semisimple Lie algebras and coherent states. Statements (11) and (2) are motivated by Theorems 2 and 11, respectively. (2) also provides an interpretation of many meanfield ground states as coherent states. This is because meanfield Hamiltonians are often expressed as operators in a small Lie algebra, in particular, operators quadratic in the creation and annihilation operators.

Statement (3) is one of the definitions of generalized coherent states. For other characterizations of generalized coherent states, see [23, 24].

Statement (4) is a version of the minimum variance principle for coherent states [57, 58]. The variance of a state $|\psi\rangle$ with respect to $\operatorname{Re}\left(\mathfrak{h}_{0}\right)$ is computed as the expectation of an "invariant uncertainty operator". For a state $|\psi\rangle$, this expectation is given by

$$
\sum_{i} x^{i} x_{i}-\sum_{i}\left\langle\psi\left|x^{i}\right| \psi\right\rangle\left\langle\psi\left|x_{i}\right| \psi\right\rangle
$$

where $\left(x_{i}\right)_{i}$ is a basis of $\operatorname{Re}\left(\mathfrak{h}_{\circ}\right)$, and $\left(x^{i}\right)_{i}$ is the dual basis with respect to the trace inner product. This is a linear function of the $\mathfrak{h}$-purity because the second sum is the negative of the purity up to a constant due to our inclusion of the identity operator.

Statement (5) is motivated by the results concerning the classical simulatability of fermionic linear optics [59, 60]. Simulatability depends crucially on the fact that the initial state preparations and the measurements outcomes can be expressed in terms of projectors in $\overline{e^{\mathfrak{h}}}$. 
Proof of Theorem 14. (2) $\Rightarrow$ (3): Let $\mathfrak{c}$ be a $\dagger$-closed Cartan subalgebra of $\mathfrak{h}_{\circ}$ containing $H$. We can perturb $H$ slightly without affecting the ground space by adding a generic element of $\mathfrak{c}$ to make sure that $H$ is generic, that is, so that the commutant of $H$ is $\mathfrak{c}$. The commutant of $H$ is the set of elements of $\mathfrak{h}_{0}$ that commute with $H$. It therefore suffices to show that ground states of generic elements of $\mathfrak{c}$ are minimum weight for an ordering of the roots. Note that for no non-zero root $\alpha$ is $\alpha(H)=0$, because otherwise $H$ is not generic. Thus we can call a root positive if $\alpha(H)>0$, and there is some simple root system for which this coincides with the definition of positive roots. A ground state is annihilated by the root spaces of $\mathfrak{h}_{0}$ that correspond to the negative roots. This implies that it is a minimum weight state.

(3) $\Rightarrow$ (2): Every minimum-weight vector $|\psi\rangle$ has minimum weight for some $\dagger$-closed Cartan subalgebra $\mathfrak{c}$ with root basis $\alpha_{1}, \ldots, \alpha_{d}$. There is a hermitian member $H$ of $\mathfrak{c}$ for which $\alpha_{k}(H)>0$ for each $k .|\psi\rangle$ is the ground state of $H$.

(2) $\Rightarrow$ (5): Let $\lambda$ be the eigenvalue of $|\psi\rangle$ for $H$. Then the desired projector is $\lim _{t \rightarrow \infty} e^{(-H+\lambda) t}$.

((5) \& (2) $\Rightarrow(3))) \Rightarrow$ (3): Let $|\psi\rangle\langle\psi|=\lim _{k} e^{-H_{k}}$, with $H_{k} \in \mathfrak{h}$. Then $|\psi\rangle\langle\psi|=$ $\lim _{k} e^{-H_{k}} e^{-H_{k}^{\dagger}}$. The operators in the limit are now hermitian, which implies that they can be written in the form $e^{-h_{k}}$, with $h_{k}$ hermitian in $\mathfrak{h}$. For sufficiently large $k$, the minimum eigenvalue of $h_{k}$ must be unique. This eigenvalue must go to zero and the eigenvalue gap $\delta_{k}$ of $h_{k}$ goes to infinity. Thus for sufficiently large $k$, the ground state $\left|\psi_{k}\right\rangle$ of $h_{k}$ is projectively well-defined. Because of $((2) \Rightarrow(3))),\left|\psi_{k}\right\rangle$ is a minimum weight state. Minimum weight states form an orbit of $e^{\operatorname{Re}(\mathfrak{h})}$, a compact set. Thus there is a cluster point $\left|\psi_{0}\right\rangle$ of the $\left|\psi_{k}\right\rangle$. It must be the case that $\left|\psi_{0}\right\rangle \propto|\psi\rangle$. Hence $|\psi\rangle$ is minimum weight.

(4) $\Rightarrow$ (11): By convexity of purity.

$(11) \Rightarrow(\sqrt[3]{3})$ : Let $\mathfrak{c}$ be the $\dagger$-closed Cartan subalgebra containing the projection of $\rho$ into $\mathfrak{h}$. We call this a supporting Cartan subalgebra of $\rho$. Let $\mathcal{H}_{\alpha}$ be the weight spaces with respect to this Cartan subalgebra. Then $\lambda$ is zero on the non-zero root spaces with respect to $\mathfrak{c}$. Since $\rho$ is a mixture of normalized superpositions of weight vectors $\left|v_{\alpha}\right\rangle \in \mathcal{H}_{\alpha}$, it follows that $\lambda\lceil\mathfrak{c}$ is a convex combination of weights. But the weights are all in the convex closure of the set of minimum weights with respect to different orderings of the roots. Extremality therefore requires that $\lambda\lceil\mathfrak{c}$ is given by a minimum weight. Let $|\psi\rangle$ be the corresponding minimum weight state. By choice of $\mathfrak{c}$, $\lambda$ is also induced by $|\psi\rangle\langle\psi|$. The density matrix $\rho$ cannot have a contribution to the mixture with different weight spaces, as otherwise, $\lambda \mid \mathfrak{c}$ is in the strict interior of the convex closure of the set of minimum weights. That $\rho=|\psi\rangle\langle\psi|$ now follows from the fact that due to irreducibility of $\mathfrak{h}$, the minimum weight spaces are one-dimensional.

Note that supporting Cartan subalgebras' weight spaces generalize the Schmidt basis used to diagonalize reduced density matrices in the bipartite setting. See Theorem 23 .

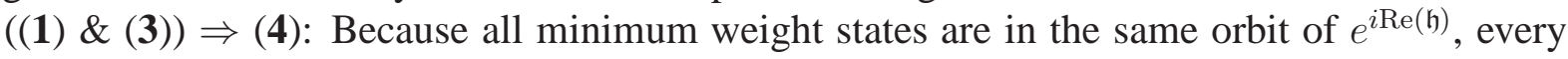
minimum weight state has the same purity. By extremality and convexity of purity, minimum weight states have maximum purity.

\section{B. Local Quantum Maps}

We can use Theorem 3 to generalize separable maps. Thus we define $\mathfrak{h}$-separable quantum maps to be those with an explicit form $\left(A_{k}\right)_{k}$ with $A_{k} \in \overline{e^{\mathfrak{h}}}$. To generalize LOCC maps, one can always return to the multipartite setting by using the fact that by semisimplicity, $\mathfrak{h}_{\circ}$ can be uniquely represented as a product of simple Lie algebras $\mathfrak{h}_{\circ}=\times_{k} \mathfrak{h}_{k}$ (see, for example, [33], $\mathrm{p}$. 
23). The state space then factors as $\otimes_{k} \mathcal{H}_{k}$, with $\mathfrak{h}_{k}$ acting on $\mathcal{H}_{k}$ only. We define $\mathfrak{h}$-LOCC maps by conditional composition of explicit $\left(\mathfrak{h}_{k}+\mathbb{C} I\right)$-separable quantum maps. This definition is more general than the usual notion of LOCC maps for multipartite systems because $\mathfrak{h}_{k}$ can be different from $\mathfrak{s l}_{n} \mathbb{C}$ or its representation $\mathfrak{h}_{k}$ need not be the first fundamental representation.

In the bipartite setting, we discussed two other ways in which LOCC maps can be characterized. One way used liftability to well defined maps of h-states. The other used restrictions on the operators based on their eigenspaces. We consider how these ideas can lead to other interesting families of quantum maps.

A subfamily of the explicit $\mathfrak{h}$-separable quantum maps is obtained by requiring that each operator lifts to $\mathfrak{h}$. Such quantum maps are called explicit $\mathfrak{h}$-liftable quantum maps. (Recall our convention for using the word "explicit".) In the bipartite setting, Theorem 7 implies that all such quantum maps are mixtures of unitaries, a small subfamily of the LOCC maps. The conclusion of Theorem $\bigoplus$ does not hold in general. For example, trivially, if $\mathfrak{h}$ consists of all operators on $\mathcal{H}$, then all quantum maps are in this family. One nice property of the family of explicit $\mathfrak{h}$-liftable quantum maps is that there is a straightforward proof of monotonicity for a large family of entanglement measures, see Theorem 29.

A family of quantum maps that includes the explicit $\mathfrak{h}$-liftable ones consists of the $\mathfrak{h}$-separable quantum maps that are liftable to $\mathfrak{h}$. In the bipartite setting, this family may be larger than the family of LOCC maps, see Problem 6. In the general setting, we pose the following problem:

Problem 15 Is the family of quantum maps obtained by conditional composition of explicit $\mathfrak{h}$ separable quantum maps that are liftable to $\mathfrak{h}$ strictly smaller than the family of $\mathfrak{h}$-separable quantum maps?

Based on Theorem 8 and its corollary, one might want to consider the family of maps consisting of binary $\mathfrak{h}$-separable quantum maps. Unfortunately, this family can be trivial in the sense that in many cases it consist of mixed unitary quantum maps only. For example, consider the spin-1 Lie algebra and suppose that $(A, B)$ is an explicit separable quantum map. We have $A, B \in e^{\mathfrak{h}}$ and $A^{\dagger} A+B^{\dagger} B=I$. The operators $A^{\dagger} A$ and $B^{\dagger} B$ are in $e^{\mathfrak{h}}$ and can be written in the form $e^{H_{A}}$ and $e^{H_{B}}$ with $H_{A}$ and $H_{B}$ in $\operatorname{Re}(\mathfrak{h})$. Thus $H_{A}=\alpha I+\vec{x} \cdot \vec{J}$. With a suitable rotation, we can assume that $H_{A}=\alpha+\beta J_{z}$. This ensures that $e^{H_{A}}$ is diagonal in the basis $|-1\rangle,|0\rangle,|1\rangle$ and has diagonal entries $e^{\alpha-\beta}, e^{\alpha}, e^{\alpha+\beta}$. It follows that $e^{H_{B}}$ is diagonal also, and hence of the same form with $\alpha^{\prime}$ and $\beta^{\prime}$. Their sum is $I$, and it can be checked that the solutions satisfy $\beta=\beta^{\prime}=0$. Hence $A$ and $B$ are proportional to unitaries.

One idea for avoiding the possible triviality of binary $\mathfrak{h}$-separable quantum maps is to use $k$-ary quantum maps. That is, consider extremal $k$-ary $\mathfrak{h}$-separable quantum maps. A quantum map is extremal if its action on density matrices is not a convex combination of other quantum maps. Because mixed unitary quantum maps are not extremal unless they are unitary, the spin-1 example shows that there may be no such extremal quantum maps for $k=2$. Let $k_{\min }$ be the minimum $k>1$ for which such quantum maps exist. Let the family of minimally generated separable quantum maps consist of explicit quantum maps obtained by conditional composition of unary or extremal $k_{\min }$-ary $\mathfrak{h}$-separable quantum maps. Because of Corollary 9 , this family is the family of LOCC maps in the bipartite setting.

Problem 16 What is the relationship between the family of minimally generated $\mathfrak{h}$-separable quantum maps, $\mathfrak{h}$-LOCC and and $\mathfrak{h}$-separable quantum maps?

Another family of quantum maps that might be interesting is obtained by adding the liftability condition to the generators of the family in the above problem. 
We now move on to considering families of $\mathfrak{h}$-separable quantum maps that are characterized by generators with large ground spaces. Based on Theorem 10, we can define a maximally $\mathfrak{h}$ unilocal operator to be an operator in $\operatorname{Re}(\mathfrak{h})$ whose ground space is maximal. These operators have a Lie algebraic characterization.

Theorem 17 Maximally $\mathfrak{h}_{0}$-unilocal operators are the ones that are proportional to a an operator of the dual basis to a simple root system of a $\dagger$-closed Cartan subalgebra of $\mathfrak{h}_{0}$.

The dual basis of a simple root system corresponds to the fundamental weights via the isomorphism induced by the Killing form. The Killing form is the symmetric bilinear form associated with the trace in the adjoint representation. The $k$ 'th fundamental weight $\lambda_{k}$ for a simple root system consisting of the roots $\alpha_{l}$ has the property that if $h_{l}=\left[x_{l}, y_{l}\right]$ with $x_{l}$ and $y_{l}$ members of the root space for $\alpha_{l}$ and for $-\alpha_{l}$, respectively, then $\lambda_{k}(h)=0$ except for $l=k$. It also has minimum length among weights satisfying this property. Fundamental weights are important because all the representations of a Lie algebra can be built from ones whose minimum weight is fundamental.

Proof of Theorem 17. Let $H \in \operatorname{Re}(\mathfrak{h})$ and choose a $\dagger$-closed Cartan subalgebra $\mathfrak{c}$ containing $H$ and an ordering of the roots such that for positive roots $\alpha, \alpha(H) \geq 0$. Let $\left(\alpha_{k}\right)_{k}$ be the simple root system for this ordering. Let $\mathcal{H}_{0}$ be the ground space of $H$. Then $\mathcal{H}_{0}$ is a union of weight spaces of $\mathfrak{c}$. By definition of the ground space, if $X$ is in the root space for a negative root, then $X \mathcal{H}_{0} \subseteq \mathcal{H}_{0}$. In particular, $\mathcal{H}_{0}$ contains the weight space for the minimum weight $\lambda_{0}$ of the chosen ordering of the roots. Furthermore, $\mathcal{H}_{0}$ consists exactly of the weights $\lambda$ such that $\lambda-\lambda_{0}$ is a positive integral combination of positive roots $\alpha$ with $\alpha\left(\mathcal{H}_{0}\right)=0$. Thus $\mathcal{H}_{0}$ is non-trivially maximal iff $\alpha_{k}\left(\mathcal{H}_{0}\right)=0$ for all but one $k=k_{0}$. Given $k_{0}$, the set of operators with this property is necessarily one-dimensional and contains one that contributes to the dual basis of the simple root system. This follows from the fact that the simple roots are a basis of the dual space of $\mathfrak{c}$.

The maximally $\mathfrak{h}$-unilocal operators fall into different classes depending on the associated fundamental weight. However, it is likely that if $\mathfrak{h}_{0}$ is simple, then the linear span of the $e^{\mathfrak{h}}$ conjugates of a given maximally $\mathfrak{h}_{0}$-unilocal operator is all of $\mathfrak{h}_{0}$. We do not know whether this holds in general, but it is certainly the case for $\mathfrak{h}_{a}$ and $\mathfrak{h}_{b}$ and $\mathfrak{g}$. This implies that if we define m-compatibility as in the bipartite setting and close under conditional composition, we might get all $\mathfrak{h}$-LOCC maps. So define a $\mathfrak{h}$-compatible family of operators as a family consisting of the $e^{\mathfrak{h}}$ conjugates of a maximally $\mathfrak{h}$-unilocal operator.

Problem 18 Does conditional composition of explicit separable quantum maps with operators from an $\mathfrak{h}$-compatible family generate the family of $\mathfrak{h}$-LOCC maps?

For now, the properties of the various families of quantum maps are largely unknown and offer a fruitful area of further investigation.

\section{Communication Complexity}

Communication complexity can be defined exactly as in the bipartite setting for any of the families of explicit quantum maps defined by conditional composition in the previous section. 


\section{Resource Scaling}

The goal is to determine what might be reasonable choices of "scaled" Lie algebras $\mathfrak{h}{ }^{\circledast n}$ acting on $\mathcal{H}^{\otimes n}$ extending the action of $\mathfrak{h}$ on each factor so as to be consistent with the corresponding picture for bipartite systems. It makes sense to require that $\mathfrak{h}{ }^{\circledast n}$ be contained in $G_{n}(\mathfrak{h})$, the set of operators $X$ with the property that if $Y$ is an operator acting as the identity on the $k$ 'th factor of $\mathcal{H}^{\otimes n}$, then the partial trace of $X Y$ onto the $k$ 'th factor is in $\mathfrak{h}$ acting on this factor. In the bipartite case, it was possible to obtain the desired $\mathfrak{h}^{\circledast n}$ by appealing to the two unilocal Lie algebras contained in $\mathfrak{h}$. We can similarly use any generating Lie subalgebras. That is, let $\mathfrak{h}$ be generated by Lie subalgebras $\mathfrak{h}_{k}$. With respect to these Lie subalgebras, we can define $\mathfrak{h}^{\circledast n}$ as the Lie algebra generated by $G_{n}\left(\mathfrak{h}_{k}\right)$. In this case, it makes sense to define $\mathfrak{h}_{k}{ }^{\circledast n}=G_{n}\left(\mathfrak{h}_{k}\right)$. At this point, we do not know to what extent this scheme is useful in analyzing the asymptotic relationships between states from the point of view of $\mathfrak{h}$. As a potentially interesting alternative, the scheme based on extensions of the permutation group discussed in the last paragraph of of Section [ID can of course be applied to any Lie algebra of operators.

\section{E. Measures of Relative Entanglement}

From the point of view of $\mathfrak{h}$, incoherent pure states of $\mathcal{H}$ look like a mixture of coherent states. This is because the $\mathfrak{h}$-state induced by an incoherent state is a proper convex combinations of pure $\mathfrak{h}$-states. However, incoherent pure states can exhibit generalized entanglement provided that it is possible to refer to operators outside of $\mathfrak{h}$. We therefore need access to observables in a larger Lie algebra. Let $\mathfrak{g} \supset \mathfrak{h}$ be a Lie algebra of operators on $\mathcal{H}$. Theorem 14 applies to $\mathfrak{g}$ as well, and in general, not all pure $\mathfrak{g}$-states are pure when restricted to $\mathfrak{h}$. Note that a $\mathfrak{g}$-state that restricts to a pure $\mathfrak{h}$-state state is necessarily pure. So it makes sense to call a pure $\mathfrak{g}$-state $\mathfrak{h}$-coherent if it restricts to a pure $\mathfrak{h}$-state.

The goal of this section is to find ways to quantify the relative entanglement of $\mathfrak{g}$-states with respect to $\mathfrak{h}$. The idea is that $\mathfrak{h}$-coherent $\mathfrak{g}$-states are not entangled, while any other pure $\mathfrak{g}$-state is definitely entangled, but the extent of entanglement depends in some way on how far the state is from being pure when restricted to $\mathfrak{h}$. Once the entanglement of pure $\mathfrak{g}$-states has been quantified, this can be extended to arbitrary $\mathfrak{g}$-states.

Let $S$ be a Schur-concave function of probability distributions. Then we can define $S(\lambda)$ for $\mathfrak{h}$-states $\lambda$ and $S\left(\lambda^{\prime} ; \mathfrak{h}\right)$ for $\mathfrak{g}$-states $\lambda^{\prime}$ as we did in Section IIE. In the bipartite setting, $S(\lambda)$ is concave as a function of $\mathfrak{h}$-states $\lambda$.

Problem 19 For which $\mathfrak{h}$ is $S$ a concave function of $\mathfrak{h}$-states?

That $S(\lambda ; \mathfrak{h})$ is a convex function of $\mathfrak{g}$-states $\lambda$ will be shown in the more general setting of convex cones, where we will also discuss the issue of monotonicity of $S$ under the various notions of generalized local quantum maps.

Another measure that can be used for quantifying generalized entanglement is based on purity. Let $p\left(\lambda^{\prime}\right)$ denote the $\mathfrak{h}$-purity of an $\mathfrak{h}$-state $\lambda^{\prime}$. We can define, for a $\mathfrak{g}$-state $\lambda$,

$$
p(\lambda ; \mathfrak{h})=\sup \left\{\sum_{k} p_{k} p\left(\lambda_{k} \uparrow \mathfrak{h}\right) \mid \lambda=\sum_{k} p_{k} \lambda_{k} \text { with } \lambda_{k} \text { pure for } \mathfrak{g}\right\}
$$

Then $p(\lambda ; \mathfrak{h})$ achieves its maximum exactly at the states that are mixtures of $\mathfrak{h}$-coherent states, and $p(\lambda ; \mathfrak{h})$ is convex in $\lambda$. Mixtures of $\mathfrak{h}$-coherent states are generalized separable states. 
Observe that for bipartite pure states, the purity is a linear function of the Renyi entropy given by $-\sum_{k} p_{k}^{2}$ where the $p_{k}$ are the eigenvalues of the reduced density matrices. In this case, the Renyi entropy can be derived from the Schur-concave function $S\left(\left(p_{k}\right)_{k}\right)=-\sum_{k} p_{k}^{2}$.

It is possible to define resource-based measures of relative entanglement as discussed at the end of Section IIE, with the caution that asymptotic versions of such measures depend on whether a useful notion of scaling for resources can been found.

One advantage of relativizing measures of entanglement by using pairs $\mathfrak{h} \subseteq \mathfrak{g}$, is that one can better investigate properties of states on systems with a hierarchy of meaningful choices for Lie algebras. Multipartite systems are examples where this situation arises. For every subset $s$ of the subsystems, there is the algebra $\mathfrak{h}_{s}$ of operators acting only on the subsystems in $s$, and the $\mathfrak{h}_{s}$ can be summed over a partition of the subsystems to obtain generalizations of $\mathfrak{h}_{l}$. These Lie algebras are ordered by inclusion. Given a state, one can, for every pair $\mathfrak{k} \subseteq \mathfrak{l}$, determine the state's generalized entanglement and use these quantities to characterize different types of states and localize the extent to which they are entangled. Other examples with multiple, physically motivated Lie algebras are discussed in Section $\mathrm{VA}$.

\section{F. Other Measures}

We mention two other types of relative entanglement measures for states that may generalize the bipartite setting. One is based on the amplitudes in a representation of a state as a superposition of coherent states, the other uses supporting Cartan subalgebras as a generalization of the Schmidt basis. Since both of them can be extended to mixed $\mathfrak{g}$-states using the construction repeatedly used above (see Equation 10), we discuss them only for pure $\mathfrak{g}$-states. Since these are induced by pure states of $\mathcal{H}$ and the relativization comes in through the extension, we define the measures for all pure states $|\psi\rangle \in \mathcal{H}$.

Let $S$ be a Schur-concave function and $|\psi\rangle$ a state that induces a pure $\mathfrak{g}$-state. We can define an entanglement measure by minimizing the $S$-complexity of $|\psi\rangle$ 's renormalized square amplitudes in writing $|\psi\rangle$ as a superposition of coherent states. Formally:

$$
S_{a}(|\psi\rangle)=\inf \left\{\left.S(\mathbf{p})\left|p_{k}=\right| \alpha_{k}\right|^{2} / \sum_{k}\left|\alpha_{k}\right|^{2} \text { where }|\psi\rangle=\sum_{k} \alpha_{k}\left|\psi_{k}\right\rangle \text { with } \mathfrak{h} \text {-coherent }\left|\psi_{k}\right\rangle .\right\}
$$

Note that by irreducibility of $\mathfrak{h}$, every state is in the span of the coherent states for $\mathfrak{h}$.

Problem 20 Is $S_{a}(|\psi\rangle)=S(|\psi\rangle)$ in the bipartite setting?

$S(|\psi\rangle)$ is defined for the bipartite setting before Theorem 13 .

A limiting case of this definition is the $\mathfrak{h}$-rank of $|\psi\rangle$ defined as the minimum number of states needed to represent $|\psi\rangle$ as a superposition of coherent states. The $\mathfrak{h}$-rank is obtained as the limit of the Schur-concave functions $S_{r}: \mathbf{p} \rightarrow \sum_{k} p_{k}^{1 / r}$ as $r \rightarrow \infty$. A special case of the $\mathfrak{h}$-rank has a long history in quantum chemistry (see, for example [61], p. 69) and has been proposed in the context of entanglement for fermions in [12], and for bosons in [13, 17].

Problem 21 What is the relationship between the amplitude-based $\left(S_{a}(|\psi\rangle)\right)$ and the convexitybased $(S(\lambda))$ measures of entanglement for pure $\mathfrak{g}$-states?

$S_{a}$ satisfies that for proper $S, S_{a}(|\psi\rangle)=0$ iff $|\psi\rangle$ is coherent for $\mathfrak{h}$. The measure $S_{C}(|\psi\rangle)$ based on supporting Cartan subalgebras does not satisfy this. To define $S_{C}(|\psi\rangle)$, let $\mathfrak{c}$ be a supporting 
Cartan subalgebra of $\mathfrak{h}$ for $|\psi\rangle\langle\psi|$. Let $P_{\alpha}$ be the projectors onto the weight spaces of $\mathfrak{c}$. We can define

$$
\left.S_{C}(|\psi\rangle)=\inf S\left(\left.\left(\left|P_{\alpha}\right| \psi\right\rangle\right|^{2}\right)_{\alpha}\right),
$$

where the minimization is over supporting Cartan subalgebras. In the generic case, there is only one supporting Cartan subalgebra. Nevertheless it would be nice if the minimization was redundant.

Problem 22 Is $\left.S\left(\left.\left(\left|P_{\alpha}\right| \psi\right\rangle\right|^{2}\right)_{\alpha}\right)$ as introduced above independent of the choice of supporting Cartan subalgebra?

Note that $S_{C}(|\psi\rangle)$ is zero for any $|\psi\rangle$ contained in a weight space for some Cartan subalgebra of $\mathfrak{h}$ and that in general, such states are not coherent for $\mathfrak{h}$. Furthermore, these weight spaces are usually not one-dimensional. Nevertheless, this measure generalizes the bipartite setting.

Theorem 23 Assume the bipartite setting with $\mathfrak{h}=\mathfrak{h}_{l}$. The weight spaces of a supporting Cartan subalgebra for $|\psi\rangle$ are the one-dimensional spaces associated with tensor products of Schmidt basis elements for each side for some choice of Schmidt basis. Hence $S_{C}(|\psi\rangle)=S(|\psi\rangle)$.

This implies that for the bipartite setting, the answer to Problem 22 is "yes".

Proof: The projection of $|\psi\rangle\langle\psi|$ into $\mathfrak{h}_{l}$ is given by $\varrho=\rho_{a} \otimes I / N_{b}+I / N_{a} \otimes \rho_{b}-I / N_{a} \otimes I / N_{b}$ where $\rho_{a}$ and $\rho_{b}$ are the respective reduced density matrices. The supporting Cartan subalgebras are the Cartan subalgebras that commute with $\varrho$. These are necessarily of the form $\mathfrak{c}_{a} \otimes I+I \otimes$ $\mathfrak{c}_{b}$, where $\mathfrak{c}_{a}$ and $\mathfrak{c}_{b}$ are $\dagger$-closed Cartan subalgebras of $\mathfrak{h}_{a}$ and $\mathfrak{h}_{b}$ that commute with $\rho_{a}$ and $\rho_{b}$, respectively. Therefore, $\mathfrak{c}_{a}\left(\mathfrak{c}_{b}\right)$ is generated by the projectors onto an orthogonal basis $B_{a}\left(B_{b}\right)$ of eigenstates of $\rho_{a}$ ( $\rho_{b}$, respectively). The associated weight spaces are one-dimensional, spanned by tensor products of members of $B_{a}$ and $B_{b}$. Because the members of $B_{a}$ and $B_{b}$ can be paired to form a Schmidt basis for $|\psi\rangle$, the result follows.

\section{THE CONVEX CONES SETTING}

Many of the notions introduced for $\dagger$-closed operator Lie algebras can be generalized even further. For example, we can work with any linear space of operators and study properties of the convex set of linear functionals induced by states. In fact, as pointed out in Section $\mathrm{VA}$, there are physically interesting cases where this may be necessary. In this section we focus on the convexity properties of the state space and investigate the extent to which local maps and measures of generalized entanglement can still be defined and retain their features.

\section{A. Convex Cones}

A convex cone $C$ is a subset of a real linear space $U$ closed under positive linear combinations. That is, if $x, y \in C$ and $p, q \geq 0$, then $p x+q y \in C$. To avoid degeneracies, we assume that $U$ is the span of $C$. Let $\dot{C}$ consist of the non-zero elements of $C$. The cone $C$ is pointed if there is a linear functional $\operatorname{tr}$ (the trace) on $U$ such $\operatorname{tr}(\dot{C})>0$. Equivalently, $C$ is pointed if $C \cap(-C)=\{0\}$. We assume that $U$ is finite dimensional and that $C$ is closed in the usual topology for $U$. For the remainder of this paper, a cone is a closed, pointed, convex cone equipped with the positive linear functional tr. For our purposes cones represent spaces of unnormalized pure and mixed 
states. In the Lie-algebraic setting, the cone is given by the set of linear functionals $\lambda \in \mathfrak{h}^{*}$ that are nonnegative multiples of $\mathfrak{h}$-states. The trace is given by evaluation of $\lambda$ at the identity $I \in \mathfrak{h}$. If $\lambda$ is induced by the matrix $\rho$, evaluation at the identity gives the usual trace, $\operatorname{tr}(\rho)$. We refer to members $x \in C$ with $\operatorname{tr}(x)=1$ as states. The pure states of $C$ are extremal states of $C$. Our assumptions on $C$ imply that every state of $C$ is a convex combination of pure states.

In the Lie-algebraic setting, we explicitly introduced a second Lie algebra $\mathfrak{g}$ when discussing measures of relative entanglement. Before we introduced such measures, $\mathfrak{g}$ was implicitly present, but was trivially associated with the set of all operators. This is because the fact that $\mathfrak{h}$-states are induced by density operators plays a crucial role. In the convex cones setting, there is no equally obvious way in which states are induced, so we explicitly introduce an outer cone $D \subseteq V$, whose states induce the states on $C$ via a linear map $\pi: V \rightarrow U$ satisfying $\pi(D)=C$, and $x \in D, \operatorname{tr}(x)=1$ implies $\operatorname{tr}(\pi(x))=1$, that is, $\pi$ is trace preserving. In the Lie algebraic setting, $\pi$ is simply the restriction map: If $\lambda$ is a $\mathfrak{g}$-state, then $\pi(\lambda)=\lambda\left\lceil\mathfrak{h} \in \mathfrak{h}^{+}\right.$. We refer to $C$ as the inner cone. If $x$ is a pure state of $C$ then $\pi^{-1}(x)$ is convex closed and its extremal states are pure states in $D$. Note that in the Lie-algebraic setting, $\pi^{-1}(x)$ for a pure $\mathfrak{h}$-state $x$ is a pure $\mathfrak{g}$-state. We define separable states of $D$ to be states in the convex closure of $\left\{\pi^{-1}(x) \mid x\right.$ is pure in $\left.C\right\}$. We denote the cone generated by the separable states of $D$ as $D_{\text {sep }}$ (this depends on $C$ ). A pure state $x$ of $D_{\text {sep }}$ satisfies that $\pi(x)$ is pure in $C$.

As we discuss the extent to which we can define suitable generalizations of various notions to the convex cones setting, it is worth keeping in mind what the two cones correspond to in the bipartite setting. In this setting, $D$ is isomorphic to the cone of positive operators on $\mathcal{H}_{a} \otimes \mathcal{H}_{b}$, with tr the usual trace functional. The trace one operators are the density matrices. $C$ is determined by the reduced density matrices. Formally, $C$ is isomorphic to the cone of operators of the form $A \otimes I / N_{b}+I / N_{a} \otimes B+\alpha I / N_{a} \otimes I / N_{b}$ with $A, B$ traceless and $A+\alpha I / N_{a}$ and $B+\alpha I / N_{b}$ positive. The connection to $\mathfrak{h}_{l}$-states is discussed in Section IIB. The map from $D$ to $C$ takes $\rho$ to $\operatorname{tr}_{b}(\rho) \otimes I / N_{b}+I / N_{a} \otimes \operatorname{tr}_{a}(\rho)-\operatorname{tr}(\rho)\left(I / N_{a} \otimes I / N_{b}\right)$.

\section{B. Local Maps}

A positive map of $D$ is a linear map $A: V \rightarrow V$ such that $A(D) \subseteq D$. The map $A$ is trace preserving if $\operatorname{tr}(x)=\operatorname{tr}(A(x))$ for all $x$. This definition corresponds to positive, but not necessarily completely positive maps in the Lie algebraic setting. Without the algebraic structure available for states, it is not possible to define a unique "tensor product" of cones, as would be required to distinguish between positive and completely positive maps [62, 63] (cited in [64]). Because of the absence of a suitable tensor product construction, we also do not have any suggestions for how to address asymptotic questions by resource scaling.

The family of positive maps of $D$ is closed under positive combinations and hence form a cone (without a trace). In the Lie-algebraic, or even the bipartite setting, the extreme points of this cone are not easy to characterize (see, for example, [64], p. 1927, 665]). However, the extreme points of the cone of completely positive maps are certainly extremality preserving in the following sense: A positive map $A$ of $D$ is extremality preserving if for all extremal $x \in D, A(x)$ is extremal. There are extremality preserving positive, not completely positive, maps. An example is partial transposition for density operators of qubits. We call a positive map that is a mixture of extremality preserving maps q-positive. It is possible to recapture the idea of complete positivity by explicitly introducing a cone representing the "tensor product" extension of $D$. This will be discussed after defining liftability. In the bipartite setting, the family of q-positive maps of $D$ is between the family of positive maps and the family of completely positive maps acting on density matrices on 
$\mathcal{H}_{a b}$.

The next step is to define a family of maps that generalizes the separable maps. Call a positive map $A$ of $D C$-separable if it is a mixture of extremality-preserving positive maps $A_{k}$ that are also extremality-preserving and positive for $D_{\text {sep }}$. In the bipartite setting, this definition includes maps such as the swap, which exchanges the two subsystems and is not separable, in addition to some non-completely positive operations. Note that if the Lie-algebraic definition of separability is used, operations like the swap are excluded because they are not in the Lie group generated by $\mathfrak{h}_{l}$ : The swap induces an exterior automorphism of $\mathfrak{h}_{l}$. From the point of view of entanglement, including the swap can make sense because it obviously does not increase entanglement.

One tool used to narrow the family of separable quantum maps was based on liftability. The definition of liftability immediately generalizes to our cones. We say that a positive map $A$ on $D$ can be lifted to $C$ if $A$ preserves the nullspace of $\pi$, or, equivalently, if there exists a positive map $A^{\prime}$ on $C$ such that $\pi(A(x))=A^{\prime}(\pi(x))$. In this case, we say that $A^{\prime}$ is the lifting of $A$ to $C$.

Using liftability, we can add more cones to try to capture the idea of complete positivity or to exclude maps like the swap. For complete positivity, introduce one more cone $E$ and positive tracepreserving map $\sigma: E \rightarrow D$ (onto). In the setting where states are defined by density matrices on a Hilbert space $\mathcal{H}$ of dimension $d, E$ represents the cone generated by density matrices on $\mathcal{H} \otimes \mathcal{H}^{\prime}$, with $\mathcal{H}^{\prime}$ of dimension at least $d^{2}$. With this cone in hand, we can try to get the completely positive maps by considering only maps that are a mixture of extremality preserving maps $A_{k}$ obtained as liftings of extremality preserving positive maps $B_{k}$ on $E$. Whether this works depends on the answer to the following problem:

Problem 24 Let $A$ be a positive map on operators of $\mathcal{H} \otimes \mathcal{H}^{\prime}$ with $\operatorname{dim}\left(\mathcal{H}^{\prime}\right) \geq \operatorname{dim}(\mathcal{H})^{2}$. Suppose that $A$ preserves the set of rank one operators and that it lifts to a map $A^{\prime}$ of operators on $\mathcal{H}$. Is $A^{\prime}$ completely positive?

To exclude the swap, it suffices to introduce cones included in $C$ to represent density matrices on $\mathcal{H}_{a}$ and $\mathcal{H}_{b}$ and require liftability to both of these cones.

The other tool used to restrict separable maps involves operators with maximal ground spaces. It is not clear how to apply this tool to the convex cone setting since the distinction between positive and negative eigenvalues is not easily recovered in the action $\rho \rightarrow A \rho A^{\dagger}$.

To be able to generate families of maps by a kind of locality preserving composition requires the idea of conditional composition based on explicit maps. An explicit positive map $\mathbf{A}$ on $D$ is given by $\mathbf{A}=\left(A_{k}\right)$ with $A_{k}$ extremality preserving positive maps. For explicit separability, the $A_{k}$ are required to be $C$-separable. In addition, we can impose the liftability condition on each $A_{k}$. We call the latter explicit $C$-liftable separable maps. The idea of Section III B to restrict the separable maps by using certain minimal explicit separable maps can be applied in the convex cones setting. However, without the strong symmetry present in the Lie-algebraic setting, the definition of $k_{\min }$ (Section [IIB) is unlikely to be as natural. However, one could investigate the families of maps obtained by replacing $k_{\min }$ by $2,3, \ldots$

Conditional composition can be used to generate a family of maps as before. One can then readily generalize communication complexity to the resulting conditionally composed maps. 


\section{Measures of Relative Entanglement}

The entanglement measures defined on the basis of a Schur-concave function $S$ are intrinsically defined using only convexity. Thus, for states $x \in C$,

$$
S(x)=\inf \left\{S(\mathbf{p}) \mid x=\sum_{k} p_{k} x_{k} \text { with } x_{k} \text { pure }\right\}
$$

and for states $x \in D$,

$$
S(x ; C)=\inf \left\{\sum_{k} p_{k} S\left(\pi\left(x_{k}\right)\right) \mid x=\sum_{k} p_{k} x_{k} \text { with } x_{k} \text { pure }\right\} .
$$

In general, $S(x)$ is not concave, though this is the case in the bipartite setting and if the set of states is a simplex. In the latter case, the expression of a point as a convex combination of extreme points is unique.

Problem 25 For which convex sets is $S(x)$ concave for all Schur-concave S?

Theorem $26 S(x ; C)$ is convex in $x$.

Proof: Let $y=p x_{1}+(1-p) x_{2}$ be a convex combination of states $x_{1}, x_{2} \in D$. We show that $S(y ; C) \leq p S\left(x_{1} ; C\right)+(1-p) S\left(x_{2} ; C\right)$, from which the theorem follows. For every way of expressing $x_{k}=\sum_{l} p_{k l} x_{k l}$ as a convex combination of pure states of $D$ we have $y=\sum_{l}\left(p p_{1 l} x_{1 l}+\right.$ $\left.(1-p) p_{2 l} x_{2 l}\right)$. Thus

$$
\begin{aligned}
S(y ; C) & \leq \sum_{l}\left(p p_{1 l} S\left(\pi\left(x_{1 l}\right)\right)+(1-p) p_{2 l} S\left(\pi\left(x_{2 l}\right)\right)\right) \quad \text { by definition, } \\
& =p \sum_{l} p_{1 l} S\left(\pi\left(x_{1 l}\right)\right)+(1-p) \sum_{l} p_{2 l} S\left(\pi\left(x_{2 l}\right)\right)
\end{aligned}
$$

The last two sums can be chosen to be arbitrarily close to $S\left(x_{1} ; C\right)$ and $S\left(x_{2} ; C\right)$.

Purity as defined in the Lie algebraic setting does not generalize to the setting of convex cones unless $C$ has a well-defined center and satisfies that all its pure states are equidistant from the center in a natural metric.

\section{Monotonicity for Explicit Liftable Maps}

A desirable property for measures of entanglement is that they are nonincreasing under the family of maps that are considered to be local.

Problem 27 For which of the families of maps that we have introduced is $S(x ; C)$ (or, more specifically, $S(x ; \mathfrak{h}))$ nonincreasing?

In the bipartite setting, it has been shown that $S\left(x ; \mathfrak{h}_{l}\right)$ is nonincreasing under LOCC maps [48]. Here we show that this is the case in the convex cones setting for the family of trace-preserving explicit liftable $C$-separable maps of cones. With the cones that arise in the bipartite setting, this family of maps includes the explicit liftable separable quantum maps. (See also Problem 24.) The monotonicity result is easy to see for the later family because in this case, the family of maps consists of mixtures of product unitaries. 
For $x \neq 0$ in a cone, define $\widehat{x}=\widehat{\Upsilon}(x)=\operatorname{tr}(x)^{-1} x$ to be the unique state proportional to $x$. If $x=0$, define $\widehat{x}=0$. We say that the function $\Upsilon: D \rightarrow \mathbb{R}$ is explicitly nonincreasing for the trace-preserving explicit positive map $\mathbf{A}=\left(A_{k}\right)_{k}$ if for extremal states $x \in D$,

$$
\Upsilon(x) \geq \sum_{k} p_{k} \Upsilon\left(\widehat{A_{k}(x)}\right)
$$

where $p_{k}=\operatorname{tr}\left(\widehat{A_{k}(x)}\right)$. The property of being explicitly nonincreasing is useful as a sufficient condition for being nonincreasing.

Lemma 28 Suppose that $S(x ; C)$ is explicitly nonincreasing for the trace-preserving explicit positive map A. Then $S(x ; C)$ is nonincreasing for $\mathbf{A}$.

The Lemma holds for any $\Upsilon$ defined from its values on pure states according to $\Upsilon(x)=$ $\inf \left\{\sum_{k} p_{k} \Upsilon\left(x_{k}\right) \mid x=\sum_{k} p_{k} x_{k}\right.$ with $x_{k}$ pure $\}$.

Proof: Let $\mathbf{A}=\left(A_{k}\right)_{k}$ with $A_{k}$ positive and write $p_{k}=\operatorname{tr}\left(A_{k}(x)\right)$ To prove the lemma, first consider an extremal $x$. Then

$$
\begin{array}{rlr}
S(\mathbf{A}(x) ; C) & =S\left(\sum_{k} A_{k}(x) ; C\right) & \\
& \leq \sum_{k} p_{k} S\left(\widehat{A_{k}(x)} ; C\right) & \text { by convexity, } \\
& \leq S(x ; C) & \text { by being explicitly nonincreasing. }
\end{array}
$$

For a nonextremal $x$, write $x=\sum_{l} q_{l} x_{l}$ with $x_{l}$ pure and $\sum_{l} q_{l} S\left(x_{l} ; C\right)$ arbitrarily close to $S(x ; C)$. Note that for pure $y, S(y ; C)=S(\pi(y))$. Then

$$
\begin{array}{rlrl}
S(\mathbf{A}(x) ; C) & =S\left(\sum_{l} q_{l} \mathbf{A}\left(x_{l}\right) ; C\right) & & \text { by linearity, } \\
& \leq \sum_{l} q_{l} S\left(\mathbf{A}\left(x_{l}\right) ; C\right) & \text { by convexity and trace preservation, } \\
& \leq \sum_{l} q_{l} S\left(x_{l} ; C\right) & & \text { by extremality of } x_{l} .
\end{array}
$$

The result now follows because the the right hand side is arbitrarily close to $S(x, C)$.

Theorem 29 If $\mathbf{A}$ is a trace-preserving explicit liftable $C$-separable map of $D$, then $S(x ; C)$ is explicitly nonincreasing under $\mathbf{A}$.

Proof: Let $\mathbf{A}=\left(A_{k}\right)_{k}$ with each $A_{k}$ liftable to $C$ and $C$-separable. Write $p_{k}=\operatorname{tr}\left(A_{k}(x)\right.$ ). Because of Lemma 28, it is sufficient to prove Inequality 17. Let $x$ be a pure state of $D$. Let $\pi(x)=\sum_{l} q_{l} y_{l}$ be a convex representation of $\pi(x)$ in terms of pure states of $C$ such that $S(\mathbf{q})$ is arbitrarily close to $S(x ; C)=S(\pi(x))$. We can find pure states $z_{l} \in D$ such that $\pi\left(z_{l}\right)=y_{l}$. Thus $x=\sum_{l} z_{l}+z$ for some $z$ with $\pi(z)=0$. With the appropriate interpretation of $A_{k}(x) / p_{k}$ when $p_{k}=0$,

$$
\begin{aligned}
& \pi\left(\widehat{A_{k}(x)}\right)=\pi\left(A_{k}(x) / p_{k}\right) \\
& =\pi\left(A_{k}\left(\sum_{l} q_{l} z_{l}+z\right) / p_{k}\right) \\
& =\sum_{l}\left(q_{l} / p_{k}\right) \pi\left(A_{k}\left(z_{l}\right)\right) \quad \text { since } A_{k} \text { preserves the nullspace of } \pi \text {, } \\
& =\sum_{l}\left(r_{l k} q_{l} / p_{k}\right) \pi\left(\widehat{A_{k}\left(z_{l}\right)}\right) \text { with } r_{l k}=\operatorname{tr}\left(A_{k}\left(z_{l}\right)\right) \text {. }
\end{aligned}
$$


Since $A_{k}$ is $C$-separable and $z_{l}$ is pure in $D_{\text {sep }}$, so is $A_{k}\left(z_{l}\right)$. Thus, by definition, $S\left(\widehat{A_{k}(x)} ; C\right)=$ $S\left(\pi\left(\widehat{A_{k}(x)}\right)\right) \leq S\left(\left(r_{l k} q_{l} / p_{k}\right)_{l}\right)$. To prove the desired inequality, bound as follows:

$$
\begin{aligned}
\sum_{k} p_{k} S\left(\widehat{A_{k}(x)} ; C\right) & \leq \sum_{k} p_{k} S\left(\left(r_{l k} q_{l} / p_{k}\right)_{l}\right) \\
& \leq S\left(\sum_{k} p_{k}\left(r_{l k} q_{l} / p_{k}\right)_{l}\right) \quad \text { by Schur concavity, } \\
& =S\left(\left(\sum_{k} r_{l k} q_{l}\right)_{l}\right) \\
& =S\left(\left(q_{l}\right)_{l}\right)=S(\mathbf{q}) \quad \text { because A is trace preserving, }
\end{aligned}
$$

which is arbitrarily close to $S(x ; C)$.

Conditional composition of trace-preserving explicit liftable $C$-separable maps preserves explicit liftability and $C$-separability. Nevertheless it is useful to know circumstances that guarantee that conditional composition preserves monotonicity of $S(x ; C)$.

Theorem 30 Suppose that $S(x ; C)$ is explicitly nonincreasing under the trace-preserving explicit extremality-preserving maps $\mathbf{A}=\left(A_{k}\right)_{k}$ and $\mathbf{B}_{k}$. Then it is explicitly nonincreasing under the conditional composition $\mathbf{E}$ of $\mathbf{A}$ followed by the $\mathbf{B}_{k}$. $\mathbf{E}$ is also an explicit extremality-preserving map.

Proof: Let $x$ be a pure state of $D$. That $\mathbf{E}$ is also an explicit extremality-preserving map is clear. Write $p_{k}=\operatorname{tr}\left(A_{k}(x)\right)$ and $q_{k l}=\operatorname{tr}\left(B_{k l}\left(A_{k}(x)\right)\right) / p_{k}$. If $p_{k}=0$, set $q_{k l}=0$. To prove Inequality 17, compute

$$
\begin{array}{rlrl}
\sum_{k l} q_{k l} p_{k} S\left(\uparrow\left(B_{k l}\left(A_{k}(x)\right)\right)\right. & ; C) & \\
& =\sum_{k l} q_{k l} p_{k} S\left(\uparrow\left(B_{k l}\left(\widehat{A_{k}(x)}\right)\right) ; C\right) & \\
& \leq \sum_{k} p_{k} S\left(\widehat{A_{k}(x)} ; C\right) & & \text { because the } \mathbf{B}_{k} \text { are explicitly non- } \\
& \leq S(x ; C) & & \text { increasing and the } A_{k}(x) \text { are extremal. }
\end{array}
$$

\section{DISCUSSION}

\section{A. Further Examples and Extensions}

The traditional setting for studies of entanglement is that of bipartite systems. Our investigation shows that the more general theory based on Lie algebras exhibits most of the features associated with bipartite entanglement, and a significant number of these features can even be found in the convex cones setting. As a result, we hope that the general theory provides new insights into bipartite entanglement and its generalizations to multipartite systems. Relativizing the idea of entanglement has the advantage of being able to immediately use the entire hierarchy of local Lie algebras and associated entanglement measures in the multipartite setting.

There are other settings where multiple, physically motivated Lie algebras occur. We give four examples of such settings. The first example involves spectrum generating algebras (SGAs). SGAs are used to determine the spectrum (eigenvalues and eigenspaces) of quantum systems. SGAs provide the starting point for one or more chains of Lie subalgebras that are used for obtaining algebraic bases of states and for expanding the Hamiltonian as a linear combination of 
invariant (Casimir) operators belonging to the chains. When such an expansion contains only invariant operators of a single algebraic chain, the system exhibits a dynamical symmetry, and the corresponding spectrum can be calculated exactly using the representation theory of Lie subalgebras. In the generic case where operators from multiple chains occur (that is, distinct dynamical symmetries coexist), the SGA approach may still make it possible to accurately represent the Hamiltonian in terms of a small number of algebraic operators. Since they were introduced in nuclear physics [66], SGA methods have been successfully applied to a variety of problems in molecular, atomic, and condensed matter physics [67]. Using the approach developed here, one could investigate the states' relationships to the families of coherent states associated with the Lie subalgebras and quantify their relative entanglement.

An example we have already mentioned as motivation for our work involves fermions in $N$ modes. In this case, in addition to the algebra of all relevant operators, there is the Lie algebra $\mathfrak{h}_{p}$ of number-preserving operators quadratic in the creation and annihilation operators. These operators can be expressed in the form $\mathbf{a}^{\dagger} M \mathbf{a}$ where $M$ is an $N \times N$ matrix. The $\mathfrak{h}_{p}$-coherent states are the Slater determinants (see, for example, [20], p. 7) and represent independent fermions. If the Lie algebra is enlarged to $\mathfrak{h}_{a}$ consisting of all operators that are homogeneous quadratic in the creation and annihilation operators, coherent states include BCS states [68], which can be thought of as describing independent fermion-pairs. Therefore, from this perspective, BCS states are unentangled. On the other hand, they have entanglement with respect to the pair $\mathfrak{h}_{p} \subset \mathfrak{h}_{a}$ of Lie algebras.

The example of fermions generalizes to anyons. Anyons as defined in quantum field theory include particles with fractional exchange statistics [69]. To apply our theory to anyons requires using features of the convex cones setting. This is because the various sets of operators quadratic in the creation and annihilation operators are Lie algebras only for fermions and bosons [70]. This was one of our motivations for extending the formalism. The convex cones can be defined as the set of linear functionals induced by states on sets of operators as before and investigated using essentially the same basic tools. Further investigation is required to determine whether special properties not available in the convex cones setting still apply to quadratic anyonic operator families.

For bosons in $N$ modes, four algebras frequently play an important role. The smallest, $\mathfrak{h}_{p l}$ consists of the operators of the form $\mathbf{a}^{\dagger} M \mathbf{a}$, where $M$ is an $N \times N$ matrix and a is the vector of annihilation operators of the $N$ modes. This algebra generates the passive linear optics operators. A second Lie algebra, $\mathfrak{h}_{s}$ is the one that generates shifts in the canonical variables associated with the modes and consists of operators at most linear in the creation and annihilation operators. The Lie algebra $\mathfrak{h}_{a l} \supseteq \mathfrak{h}_{p l}+\mathfrak{h}_{s}$ consisting of all operators that are at most quadratic in the annihilation and creation operators is the algebra that generates all linear optics operators. Finally, there is the algebra of all relevant operators. The usual coherent states of optics and harmonic oscillators are the $\mathfrak{h}_{s}$-coherent states.

Although much of our proposal can be applied to the example of bosons, caution is required in generalizing the finite dimensional theory to the infinite dimensional state spaces of bosonic modes. In addition, algebras like $\mathfrak{h}_{s}$ are not semisimple or reductive, requiring an extension of the theory, as can be done for the theory of coherent states [23, 24].

\section{B. Relevance to Condensed Matter Physics}

Entanglement, and our generalizations of it, may be important in the understanding of physical phenomena. For example, the concept of "quantum phase transitions" [71] involves a qualitative 
change in the behavior of correlation functions at zero temperature, i.e. in a pure ground state, as parameters in a system's Hamiltonian are varied. In some cases an order parameter is associated to the transition, in others a topological order. Since classical pure states cannot exhibit correlations, this is an essentially quantum phenomenon. Moreover, the presence of correlations between subsystems in a pure state can serve as a definition of entanglement, so quantum phase transitions might be considered to be due to a qualitative change in the nature of entanglement. Therefore, quantifying and classifying entanglement may help characterizing a quantum phase transition. Can measures of entanglement distinguish between a broken symmetry and a topological phase transition? Can one classify quantum critical points? It is essential in this regard to have a notion of entanglement that need not make reference to locality or subsystems. Whether the correlation functions that best characterize a given phase transition are those of distinguishable subsystems (say, lattice sites) or some other kind of correlations (say, two-particle correlation functions for systems of indistinguishable particles) may determine whether standard entanglement, or instead some generalization of it, provides appropriate concepts. Even standard entanglement is relative to a distinguished factorization of a total Hilbert space into "local" ones, though this is usually unproblematic in quantum information settings. In other settings, such as many-body condensedmatter systems, different factorizations may occur on a more equal footing as "global" transformations typically play a natural role. Thus a system of interacting bosons or fermions on a lattice may be viewed in terms of a factorization of the state space into distinguishable lattice sites, but the Fourier transformation from position modes to momentum modes may provide an alternative factorization; and it may also be that for some problems, correlations between particles, rather than modes, are relevant, taking us beyond the distinguishable-subsystems framework of standard entanglement theory.

The introduction of "quasiparticles", or transformations such as the Jordan-Wigner transformation [72, 73], may further alter the algebraic language we use to analyze the system; our motivation for such transformations may be mathematical (easier solvability in one algebraic language than in another) or physical (one algebra better exhibits the physical structure of the system's dynamics, or of our interactions with it). In either case, the coherent states formalism is often known to be useful, and tools and concepts from quantum information theory, such as generalized entanglement measures, generalized LOCC and asymptotics may help as well. Initial work in the direction of connecting the information theoretic approach to entanglement to condensed matter can be found in [74, 75, 76].

To give a more explicit example, Landau quasiparticles refer to those dressed particles of the original interacting system that weakly interact as a result of transferring most of the real interactions into the properties of the quasiparticles themselves. As a result, these quasiparticles may be qualitatively different from the original particles, an example of which is provided by the composite fermions in the quantum Hall setup [77]. But how do we construct those quasiparticles? Weak interactions can be related to weak correlations and, therefore, weak generalized entanglement. If one can re-express the original problem in a language such that the Hamiltonian operator belongs to the quadratic expressions in the language's generating operators (for example, creation and annihilation operators) then we know that the quasiparticles are non-interacting. Otherwise, we need to quantify the degree of "entanglement" (in the ground state, say) to determine whether the particles generated by the language interact sufficiently weakly to behave as true quasiparticles. The use of hierarchical languages may help to address this issue [78]. 


\section{Conclusion}

We have outlined a program whose goal is to tie together the theory of entanglement and the theory of coherent states. We implemented the first few steps of this program starting with the observation that fundamental concepts of the theory of coherent states coincide with concepts from the theory of entanglement. We extended this observation by providing general definitions of the key information-theoretic notions in entanglement theory. In particular, we introduced several classes of quantum maps to the Lie algebraic setting appropriate for coherent state theory that generalize the idea of separable maps for multipartite systems and approach LOCC. The numerous open problems attest to the richness of this program.

After noting that many of the notions that we generalized can, to some extent, be stated even more generally in the context of convex cones, we made this explicit by investigating appropriate definitions for convex cones. Except for the convex cones arising as spaces of linear functionals on operator families induced by states, most such convex cones are not physically relevant. Nevertheless, they help us appreciate what aspects of the various models are required in order to investigate different properties of generalized entanglement and their information-theoretic implications.

The main conclusion of our program so far is that conventional entanglement is a special case of a much more general theory with many of the same features. Furthermore, it is clear that entanglement is a relative property of states, requiring that states that are mixed from one perspective can be pure from other, more powerful perspectives. Once this relativity is recognized, it is possible to investigate relative entanglement of states when many physically motivated perspectives coexist. Examples include multipartite systems, condensed matter systems, and systems whose dynamics is described by the chain of Lie algebras associated with a dynamical symmetry or a spectrum generating algebra.

\section{APPENDIX A: COMPARISON OF THE SETTINGS FOR GENERALIZED ENTANGLEMENT}

The following table shows the three settings as generalizations of the bipartite setting. 


\begin{tabular}{|c|c|c|c|}
\hline & Bipartite systems & Lie algebras & Cones \\
\hline Structure: & \begin{tabular}{|l|}
$\mathcal{H}_{a} \otimes \mathcal{H}_{b}$, a tensor \\
product of two Hilbert \\
spaces.
\end{tabular} & $\begin{array}{l}\{I\} \subseteq \mathfrak{h} \subseteq \mathfrak{g}, \dagger \text {-closed Lie algebras } \\
\text { of operators on a Hilbert space } \mathcal{H}\end{array}$ & $\begin{array}{l}\text { Closed, convex cones } C \subseteq D \text { with } \\
\text { traces, and } \pi: D \rightarrow C \text { a linear, } \\
\text { trace-preserving, map onto } C \text {. }\end{array}$ \\
\hline States: & $\begin{array}{l}\text { Full or reduced density } \\
\text { matrices. }\end{array}$ & $\begin{array}{l}\text { Linear functionals on } \mathfrak{h} \text { or } \mathfrak{g} \text { induced } \\
\text { by density matrices. }\end{array}$ & Trace-one elements of $C$ or $D$. \\
\hline $\begin{array}{l}\text { Specialization } \\
\text { to bipartite } \\
\text { systems: }\end{array}$ & & $\begin{array}{l}\mathfrak{h}=\{A \otimes I+I \otimes B\}, \mathfrak{g} \text { is the set of } \\
\text { all operators on } \mathcal{H}_{a} \otimes \mathcal{H}_{b} .\end{array}$ & $\begin{array}{l}C \simeq\{(A, B) \mid A(B) \text { positive on } \\
\left.\mathcal{H}_{a}\left(\mathcal{H}_{b}\right)\right\}, D \simeq\{C \mid C \text { positive on } \\
\mathcal{H}\}, \pi(C)=\left(\operatorname{tr}_{a}(C), \operatorname{tr}_{b}(C)\right) \text {. }\end{array}$ \\
\hline $\begin{array}{l}\text { Specialization } \\
\text { to Lie algebras: }\end{array}$ & & & $\begin{array}{l}C(D) \text { consist of the linear } \\
\text { functionals induced on } \mathfrak{h}(\mathfrak{g}) \text { by } \\
\text { positive } \rho \text { on } \mathcal{H} \text { as } x \rightarrow \operatorname{tr}(\rho x) . \pi \text { is } \\
\text { the restriction map. }\end{array}$ \\
\hline $\begin{array}{l}\text { Distinguished } \\
\text { pure states: }\end{array}$ & Product pure states. & $\begin{array}{l}\text { Coherent (or, equivalently, pure) } \\
\mathfrak{h} \text {-states. }\end{array}$ & $\begin{array}{l}\text { States } x \in D \text { such that } \pi(x) \text { is pure } \\
\text { in } C \text {. }\end{array}$ \\
\hline $\begin{array}{l}\text { Distinguished } \\
\text { mixed states: }\end{array}$ & Separable states. & $\begin{array}{l}\text { Convex combinations of } \mathfrak{g} \text {-states that } \\
\text { restrict to coherent } \mathfrak{h} \text {-states. }\end{array}$ & $\begin{array}{l}\text { The cone } D_{\text {sep }} \text { of separable states in } \\
D \text { consisting of convex combinations } \\
\text { of states } x \in D \text { such that } \pi(x) \text { is pure } \\
\text { in } C \text {. }\end{array}$ \\
\hline $\begin{array}{l}\text { Pure state } \\
\text { entanglement } \\
\text { measures: }\end{array}$ & $\begin{array}{l}\text { Von-Neumann entropy } \\
\text { for pure states. } \\
\text { Unilateral purity. }\end{array}$ & $\begin{array}{l}S \text { Schur concave, } \lambda \text { an } \mathfrak{h} \text {-state: } S(\lambda) \\
=\inf \left\{S(\mathbf{p}) \mid \lambda=\sum_{k} p_{k} \lambda_{k} \text { with } \lambda_{k}\right. \\
\left.\mathfrak{h} \text {-coherent, } p_{k} \geq 0\right\} . \\
\mathfrak{h} \text {-purity. } \\
\text { Measures based on amplitudes } \\
\left(S_{a}(\lambda)\right) \text { and supporting Cartan } \\
\text { subalgebras }\left(S_{C}(\lambda)\right) \text {. }\end{array}$ & $\begin{array}{l}\text { For } x \text { a pure state in } C, S(x)= \\
\text { inf }\left\{S(\mathbf{p}) \mid x=\sum_{k} p_{k} x_{k} \text { with } x_{k}\right. \\
\left.\text { pure, } p_{k} \geq 0\right\}\end{array}$ \\
\hline $\begin{array}{l}\text { Mixed state } \\
\text { entanglement } \\
\text { measures: }\end{array}$ & $\begin{array}{l}\text { Given pure state } \\
\text { entanglement measure } \\
S: S(\rho)= \\
\inf \left\{\sum_{k} p_{k} S\left(\rho_{k}\right) !\right. \\
\sum_{k} p_{k} \rho_{k}=\rho, \rho_{k} \text { is a } \\
\text { pure product state, } \\
\left.p_{k} \geq 0\right\} .\end{array}$ & $\begin{array}{l}\text { Given an } \mathfrak{h} \text {-state measure } S \text { and a } \\
\mathfrak{g} \text {-state } \lambda, S(\lambda)=\inf \left\{\sum_{k} p_{k} S\left(\lambda_{k}\right)\right. \\
\sum_{k} p_{k} \lambda_{k}=\lambda, \lambda_{k} / \mathfrak{h} \text { is coherent, } \\
\left.p_{k} \geq 0\right\}\end{array}$ & $\begin{array}{l}\text { Given a } C \text {-measure } S, x \text { a state in } D \text {. } \\
S(x)=\inf \left\{\sum_{k} p_{k} S\left(\pi\left(x_{k}\right)\right) \mid\right. \\
\sum_{k} p_{k} x_{k}=x, \pi\left(x_{k}\right) \text { is pure, } \\
\left.p_{k} \geq 0\right\} .\end{array}$ \\
\hline $\begin{array}{l}\text { Properties of } \\
\text { entanglement } \\
\text { measures: }\end{array}$ & $\begin{array}{l}\text { Convex. Monotone } \\
\text { under LOCC. }\end{array}$ & $\begin{array}{l}\text { Convex. Monotone under explicit } \\
\text { liftable separable quantum maps. }\end{array}$ & $\begin{array}{l}\text { Convex. Monotone under } \\
\text { trace-preserving explicit liftable } \\
C \text {-separable maps of } D . \\
\end{array}$ \\
\hline \begin{tabular}{|l|} 
Maximally \\
entangled \\
states:
\end{tabular} & Bell states. & See [28]. & Undefined. \\
\hline $\begin{array}{l}\text { Non-classicality } \\
\text { of entangled } \\
\text { states: }\end{array}$ & Bell inequalities. & See [28]. & Undefined. \\
\hline Hierarchies: & $\begin{array}{l}\text { Add the unilateral } \\
\text { algebras. }\end{array}$ & $\begin{array}{l}\text { Arbitrary family of operator Lie } \\
\text { algebras ordered by inclusion. }\end{array}$ & $\begin{array}{l}\text { Arbitrary family of cones, partially } \\
\text { ordered by trace-preserving onto } \\
\text { maps. }\end{array}$ \\
\hline
\end{tabular}




\begin{tabular}{|c|c|c|c|}
\hline & Bipartite systems & Lie algebras & Cones \\
\hline $\begin{array}{l}\text { Local unitary } \\
\text { operators: }\end{array}$ & $\begin{array}{l}\text { Product unitary } \\
\text { operators. }\end{array}$ & $e^{i \operatorname{Re}(\mathfrak{h})}$. & $\begin{array}{l}\text { Positive linear isomorphism } \\
f: D \rightarrow D \text { such that } \pi f=\tilde{f} \pi \text { for } \\
\text { some isomorphism } \tilde{f}: C \rightarrow C \text {. } \\
\text { Caution: Defs of local maps do not } \\
\text { always specialize to the } \\
\text { corresponding defs for Lie algebras. }\end{array}$ \\
\hline Local operators: & Product operators. & $\overline{e^{\mathfrak{h}}}$. & $\begin{array}{l}C \text {-product maps: } \\
\text { Extremality-preserving positive maps } \\
f: D \rightarrow D \text { that preserve extremality } \\
\text { in } D_{\text {sep }} \text { also. }\end{array}$ \\
\hline Separable maps: & $\begin{array}{l}\rho \rightarrow \sum_{k} A_{k} \rho A_{k}^{\dagger} \\
\text { where the } A_{k} \text { are } \\
\text { product operators. }\end{array}$ & $\begin{array}{l}\rho \rightarrow \sum_{k} A_{k} \rho A_{k}^{\dagger}, \text { where } A_{k} \in \overline{e^{\mathfrak{h}}} \text {. } \\
\text { Caution: Defs of local maps do not } \\
\text { always specialize to the } \\
\text { corresponding defs for bipartite } \\
\text { systems. }\end{array}$ & $\begin{array}{l}C \text {-separable maps: } x \rightarrow \sum_{k} A_{k}(x), \\
\text { where the } A_{k} \text { are } C \text {-product maps }\end{array}$ \\
\hline $\begin{array}{l}\text { Unilocal } \\
\text { operators: }\end{array}$ & $A \otimes I, I \otimes A$ & $\begin{array}{l}\text { Operators of } \mathfrak{h} \text { with maximal ground } \\
\text { spaces? Operators whose action lifts } \\
\text { to } \mathfrak{h} \text {-states? }\end{array}$ & $C$-product maps of $D$ that lift to $C$ ? \\
\hline $\begin{array}{l}\text { Compatible } \\
\text { families of } \\
\text { one-sided local } \\
\text { operators: }\end{array}$ & $\begin{array}{l}\text { Operators acting on } \\
\text { the same subsystem. }\end{array}$ & $\begin{array}{l}\text { Operators conjugate under } e^{\mathfrak{h}} \text { to one } \\
\text { with maximal ground spaces? }\end{array}$ & Undefined. \\
\hline LOCC: & $\begin{array}{l}\text { Monoid generated by } \\
\text { conditional } \\
\text { composition of } \\
\text { explicit unilocal } \\
\text { quantum maps. }\end{array}$ & $\begin{array}{l}\text { Monoid generated by conditional } \\
\text { composition of explicit quantum } \\
\text { maps consisting of compatible } \\
\text { families? } \\
\text { Monoid generated by conditional } \\
\text { composition of explicit liftable } \\
\text { separable quantum maps? } \\
\ldots\end{array}$ & $\begin{array}{l}\text { Monoid generated by conditional } \\
\text { composition of trace-preserving } \\
\text { explicit liftable } C \text {-separable maps? }\end{array}$ \\
\hline $\begin{array}{l}\text { Communication } \\
\text { complexity: }\end{array}$ & \multicolumn{3}{|c|}{ Defined in terms of outcome probabilities in each step of a conditional composition. } \\
\hline \begin{tabular}{|l|} 
Known \\
monotonicity of \\
entanglement \\
results:
\end{tabular} & Under LOCC maps. & $\begin{array}{l}\text { Under explicitly liftable separable } \\
\text { quantum maps. }\end{array}$ & $\begin{array}{l}\text { Under trace-preserving explicit } \\
\text { liftable } C \text {-separable maps. }\end{array}$ \\
\hline $\begin{array}{l}\text { Resource } \\
\text { scaling: }\end{array}$ & $\begin{array}{l}\text { By tensor product, } \\
\text { preserving orientation } \\
\text { of the bipartition. }\end{array}$ & $\begin{array}{l}\text { Grow Lie algebras over tensor } \\
\text { products of } \mathcal{H} \text { using partial traces. } \\
\text { May require additional structure? }\end{array}$ & Undefined. \\
\hline
\end{tabular}




\section{Acknowledgments}

We thank Cristian Batista for the original motivation for investigating entanglement in a subsystem-independent way by pointing out that Slater determinants should be considered unentangled. We also thank the other "coherent tangles," in particular Jim Gubernatis, Leonid Gurvits, Rolando Somma and Wojciech Zurek for stimulating discussions. We acknowledge support from the DOE (contract W-7405-ENG-36).

[1] J. S. Bell, Physics 1, 195 (1964).

[2] J. S. Bell, Speakable and Unspeakable in Quantum Mechanics (Cambridge Univ. Press, Cambridge, UK, 1993).

[3] J. F. Clauser and A. Shimony, Rep. Progr. Phys. 41, 1881 (1978).

[4] P. G. A. Aspect and G. Roger, Phys. Rev. Lett. 47, 460 (1981).

[5] M. A. Rowe, D. Kielpinski, V. Meyer, C. A. Sackett, W. M. Itano, C. Monroe, and D. J. Wineland, Nature 409, 791 (2001).

[6] C. H. Bennett, G. Brassard, C. Crepeau, R. Jozsa, A. Peres, and W. Wootters, Phys. Rev. Lett. 70, 1895 (1993).

[7] R. Raz, in Proceedings of the 31st Annual ACM Symposium on the Theory of Computation (STOC) (ACM Press, El Paso, Texas, 1999), pp. 358-367.

[8] H. Buhrman, R. Cleve, and W. V. Dam, SIAM J. Comp. 30, 1829 (2000).

[9] K. Grobe, K. Rzazewski, and J. H. Eberly, J Phys B 27, L503 (1994).

[10] M. Y. Ivanov, D. Bitouk, and K. Rzazewski, Physical Review A 52, 149 (1995).

[11] J. Schliemann, D. Loss, and A. H. MacDonald, Physical Review B 63, 085311 (1999).

[12] J. Schliemann, J. I. Cirac, M. Kus, M. Lewenstein, and D. Loss, Physical Review A 64, 022303 (2001).

[13] R. Paskauskas and L. You (2001), quant-ph/0106117.

[14] P. Zanardi (2001), quant-ph/0104114.

[15] P. Zanardi and X. Wang (2002), quant-ph/0201028.

[16] Y. Shi (2002), quant-ph/0204058.

[17] K. Eckert, J. Schliemann, D. Bruss, and M. Lewenstein, Annals of Physics 299, 88 (2002).

[18] B. Yurke and D. Stoler, Phys. Rev. Lett. 57, 13 (1986).

[19] B. Yurke and D. Stoler, Physica C 151, 298 (1988).

[20] J.-P. Blaizot and G. Ripka, Quantum Theory of Finite Systems (The MIT Press, Cambridge, Mass., 1986).

[21] P. Zanardi, Phys. Rev. Lett. 87, 077901/1 (2001).

[22] R. Gilmore, Rev. Mex. de Fís. 23, 143 (1974).

[23] A. Perelomov, Generalized coherent states and their applications (Springer, Berlin/Heidelberg/New York, 1985).

[24] W.-M. Zhang, D. H. Feng, and R. Gilmore, Reviews of Modern Physics 62, 867 (1990).

[25] D. Giulini, E. Joos, C. Kiefer, J. Kupsh, I.-O. Stamatescu, and H. D. Zeh, Decoherence and the Appearance of a Classical World in Quantum Theory (Springer Verlag, Berlin, 1996).

[26] A. Peres, Quantum Theory: Concepts and Methods (Kluwer Academic, Dordrecht, 1993).

[27] C. Cohen-Tannoudji, B. Diu, and F. Laloë, Quantum Mechanics (John Wiley and Sons, New York, 1977).

[28] A. Klyachko (2002), quant-ph/0206012. 
[29] M. A. Nielsen and I. L. Chuang, Quantum Computation and Quantum Information (Cambridge University Press, 2001).

[30] M. B. Plenio and V. Vedral, Contemporary Physics 39, 431 (1998).

[31] C. Brukner, M. Zukowski, and A. Zeilinger (2001), quant-ph/0106119.

[32] M. Lewenstein, D. Bruss, J. I. Cirac, B. Kraus, M. Kus, J. Samsonowicz, A. Sanpra, and R. Tarrach, J. Mod. Opt. 47, 2481 (2000).

[33] J. E. Humphreys, Introduction to Lie algebras and representation theory, no. 9 in Graduate Texts in Mathematics (Springer, New York, 1972).

[34] V. S. Varadarajan, Lie groups, Lie algebras, and their representations, no. 102 in Graduate Texts in Mathematics (Springer, New York, 1984).

[35] W. Fulton and J. Harris, Representation Theory: a first course, no. 129 in Graduate Texts in Mathematics (Springer, New York, 1991).

[36] A. Knapp, Lie Groups: Beyond an Introduction (Birkhäuser, Boston and Basel and Berlin, 1996), (second printing, 2001).

[37] R. Gilmore, Lie Groups, Lie Algebras, and Some of Their Applications (Wiley, New York, 1973).

[38] B. G. Wybourne, Classical Groups for Physicists (Wiley, New York, 1974).

[39] J. F. Cornwell, Group Theory in Physics (Academic Press, London, 1989).

[40] J. Fuchs and C. Schweigert, Symmetries, Lie Algebras, and Representations, Cambridge Monographs on Mathematical Physics (Cambridge Univ. Press, Cambridge, England, 1997).

[41] H. Georgi, Lie Algebra in Particle Physics (Perseus Books, Reading, Mass, 1999).

[42] J. Hilger, K. H. Hoffman, and J. D. Lawson, Lie Groups, Convex Cones, and Semigroups (Clarendon Press, Oxford, 1989).

[43] A. Barvinok (2002), prepared forthe AMS Graduate Studies in Mathematics series, currently available at http://www.math.lsa.umich.edu/ barvinok/courses.html.

[44] R. F. Werner, Phys. Rev. A 40, 4277 (1989).

[45] C. H. Bennett, D. P. DiVincenzo, C. A. Fuchs, T. More, E. Rains, P. W. Shor, J. A. Smolin, and W. K. Wootters, Physical Review A 59, 1070 (1999).

[46] H. Ollivier and W. H. Zurek, Phys. Rev. Lett. 88, 017901/1 (2002).

[47] A. Abragam, Principles of Nuclear Magnetism (Clarendon Press, Oxford, England, 1961).

[48] G. Vidal, J Mod Opt 47, 355 (2000).

[49] C. H. Bennett, S. Popescu, D. Rohrlich, J. A. Smolin, and A. V. Thapliyal, Phys. Rev. A 63, 012307/1 (2001).

[50] W. Dür, G. Vidal, and J. I. Cirac (2001), quant-ph/0112124.

[51] M. J. Donald, M. Horodecki, and O. Rudolph (2001), quant-ph/0105017.

[52] R. Bhatia, Matrix Analysis (Springer, New York, 1997).

[53] H. Minc, Permanents (Addison-Wesley, Reading, Mass, 1978).

[54] C. H. Bennett, D. P. DiVincenzo, J. A. Smolin, and W. K. Wootters, Physical Review A 54, 3824 (1996).

[55] C. Bennett and P. Shor, IEEE Tr. Inf. Th. 44, 2724 (1998).

[56] J. Dixmier, Enveloping Algebras (Am. Math. Soc., Rhode Island, 1996), reprint of 1977 English translation.

[57] R. Delbourgo, Journal of Physics A 10, 1837 (1977).

[58] R. Delbourgo and J. R. Fox, Journal of Physics A 10, L232 (1977).

[59] B. M. Terhal and D. P. DiVincenzo (2001), quant-ph/0108010.

[60] E. Knill, Tech. Rep. LAUR-01-4472, Los Alamos National Laboratory (2001), quant-ph/0108033.

[61] R. McWeeny, Methods of Molecular Quantum Mechanics (Academic Press, London, 1992). 
[62] I. Namioka and R. Phelps, Pacific J. Math. 9, 469 (1969).

[63] G. Wittstock, in Foundations of quantum mechanics and ordered linear spaces, edited by H. Neumann (Springer, New York and Berlin and Heidelberg, 1974), Springer Lecture Notes in Physics.

[64] A. Wilce, Int. J. Theor. Phys. 31, 1915 (1992).

[65] L. Gurvits (2002), quant-ph/0201022.

[66] F. Iachello and A. Arima, The Interacting Boson Model (Cambridge Univ. Press, Cambridge, UK, 1987).

[67] A. Barut, A. Bohm, and Y. Ne'eman, Dynamical Groups and Spectrum Generating Algebras (World Scientific, Singapore, 1988), (two volumes).

[68] J. R. Schrieffer, Theory of Superconductivity (Perseus Books, Reading, MA, 1964).

[69] C. D. Batista and G. Ortiz (2002), cond-mat/0207106.

[70] A. Lerda and S. Sciuto, Nuc. Phys. B 401, 613 (1993).

[71] G. A. Sachdev, Quantum phase transitions (Cambridge University Press, Cambridge, 2001).

[72] P. Jordan and E. Wigner, Zeit. für Phys. 47, 631 (1928).

[73] C. D. Batista and G. Ortiz, Phys. Rev. Lett. 86, 1082 (2001).

[74] T. J. Osborne and M. A. Nielsen (2001), quant-ph/0109024.

[75] T. J. Osborne and M. A. Nielsen (2002), quant-ph/0202162.

[76] A. Osterloh, L. Amico, G. Falci, and R. Fazio, Nature 416, 608 (2002).

[77] J. K. Jain and R. K. Kamilla, in Composite Fermions (World Scientific, Singapore, 1998).

[78] C. Batista, G. Ortiz, and J. E. Gubernatis, Physical Review B 65, 180402(R) (2002). 